\title{
Multiple Bottlenecks in Hierarchical Control of Action Sequences: What Does "Response Selection" Select in Skilled Typewriting?
}

\author{
Motonori Yamaguchi, Gordon D. Logan, and Vanessa Li \\ Vanderbilt University
}

\begin{abstract}
Does response selection select words or letters in skilled typewriting? Typing performance involves hierarchically organized control processes: an outer loop that controls word level processing, and an inner loop that controls letter (or keystroke) level processing. The present study addressed whether response selection occurs in the outer loop or the inner loop by using the psychological refractory period (PRP) paradigm in which Task1 required typing single words and Task2 required vocal responses to tones. The number of letters (string length) in the words was manipulated to discriminate selection of words from selection of keystrokes. In Experiment 1, the PRP effect depended on string length of words in Task1, suggesting that response selection occurs in the inner loop. To assess contributions of the outer loop, the influence of string length was examined in a lexical-decision task that also involves word encoding and lexical access (Experiment 2), or to-be-typed words were preexposed so outer-loop processing could finish before typing started (Experiment 3). Response time for Task2 (RT2) did not depend on string length with lexical decision, and RT2 still depended on string length with typing preexposed strings. These results support the inner-loop locus of the PRP effect. In Experiment 4, typing was performed as Task2, and the effect of string length on typing RT interacted with stimulus onset asynchrony superadditively, implying that another bottleneck also exists in the outer loop. We conclude that there are at least two bottleneck processes in skilled typewriting.
\end{abstract}

Keywords: hierarchical control, dual-tasking, response selection, typing, typewriting

Many skills are organized hierarchically. Hierarchical organization makes performance of the skill easier for the practitioner (Lashley, 1951; Miller, Galanter \& Pribram, 1960), but it makes psychological analysis of the skill harder for the theorist (Vallacher \& Wegner, 1987). The level at which elementary processes like response selection are performed is ambiguous: We could be calling a friend or pressing keys on our cell phones. This article investigates the level at which response selection occurs in skilled typewriting, which is a common example of a hierarchically organized skill (Fendrick, 1937; Logan \& Crump, 2011; Shaffer, 1975a; Sternberg, Knoll, \& Turock, 1990). Typewriting involves control of rapid, sustained action sequences that express language, so the action sequences are structured hierarchically, from stories to sentences to words to keystrokes (Vallacher \& Wegner, 1987). In the present research, we asked whether the "responses" that are selected in typewriting are words or keystrokes (letters). We answered the question with a dual-task procedure called the psychological refractory period (PRP) paradigm (Pashler, 1994a; Wel-

This article was published Online First November 12, 2012.

Motonori Yamaguchi, Gordon D. Logan, and Vanessa Li, Department of Psychology, Vanderbilt University.

We thank Hal Pashler, Iring Koch, and James Enns for valuable comments on a draft of the article. This research was supported by grant number BCS 0957074 from the National Science Foundation.

Correspondence concerning this article should be addressed to Motonori Yamaguchi or Gordon D. Logan, Department of Psychology, Vanderbilt University, Nashville, TN 37240. E-mail: motonori.yamaguchi@vanderbilt .edu or gordon.logan@vanderbilt.edu ford, 1952), in which skilled typists typed a visually presented word and produced a vocal response to an auditory stimulus. We varied the number of letters in the word to dissociate the number of words (one per trial) from the number of keystrokes (three, four, or five per trial). If typists select words, then the second task should be delayed by the same amount regardless of string length. If typists select keystrokes, then the second task should be delayed more the larger the number of keystrokes (see also Pashler, 1994c).

\section{Selecting a Word or a Letter?}

Response selection is a process that chooses one action from a set of several alternatives. The process was once called an "expression of the will" (Donders, 1986/1969, p. 418), and it is often defined in terms of the experimental variables that are thought to influence its duration (stimulus-response compatibility, the number of alternative responses, etc.; Sternberg, 1969b). Most cognitive tasks studied in laboratories require a single action to each stimulus event, so there is not much ambiguity as to what constitutes a response (e.g., pressing a key or uttering a letter name). However, it is not clear what constitutes a response when task are organized hierarchically (Vallacher \& Wegner, 1987). In typewriting, for example, stimuli are hierarchically organized, from a story to sentences to words and to letters, and action selection occurs at each level of the hierarchy. An action at any of these levels could be considered a response. In cognitive psychology, response selection has a special status; researchers consider the process to be a central component by which cognitive resources are consumed, producing dual-task interference when multiple tasks must be performed concurrently (McCann \& Johnston, 1992; Pashler, 1984; Tombu \& Jolicœur, 2003; Welford, 1952). The main aim of 
the present study is to examine what constitutes response selection in skilled typewriting by looking at the locus of dual-task interference.

There are good theoretical and empirical reasons to expect typists to select words and to expect they select keystrokes. Theoretical justification is found in hierarchical theories of typing performance (Logan \& Crump, 2011; see also Rumelhart \& Norman, 1982; Shaffer, 1975a; Sternberg et al., 1990). According to these theories, skilled typing is controlled by two nested control loops, an outer loop and an inner loop (see Figure 1). The outer loop comprehends or generates sentences and produces a series of words to be typed. The inner loop receives words one at a time from the outer loop, decomposes them into letters, and implements the corresponding keystrokes. The two hierarchical loops are dissociable empirically, and studies indicate that the two loops operate autonomously (see Logan \& Crump, 2011, for a review). Response selection could occur in the outer loop (selecting words) or in the inner loop (selecting keystrokes) or in both loops.

Several lines of empirical evidence suggest that words are the responses that are selected. First, performance deteriorates when the letters in a word are scrambled, whereas performance is intact when words in a sentence are scrambled (Fendrick, 1937; Hershman \& Hillix, 1965; Shaffer \& Hardwick, 1968). Second, preview of letters to be typed increases the speed of copy typing, but the speed of typing remains slow when copying strings of random letters as compared to copying normal words (Fendrick, 1937; Shaffer, 1973). Third, presenting a word primes the keystrokes required to type the word, regardless of the position of the corresponding letter in the word, but presenting a string of random letters does not (Crump \& Logan, 2010a; see also Logan, 2003;

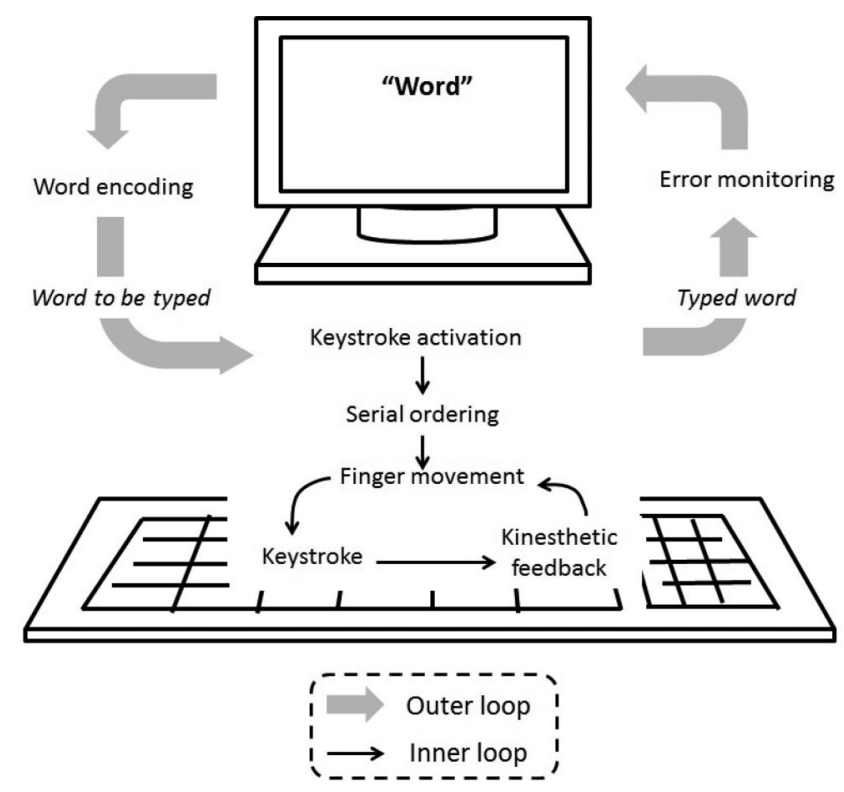

Figure 1. Schematic illustration of the outer loop and inner loop hypothesized in the two-loop theory of skilled typewriting. Adapted from "Speed-accuracy tradeoff in skilled typewriting: Decomposing the contribution of hierarchical control loops," by M. Yamaguchi, J. C. M. Crump, and G. D. Logan (in press), Journal of Experimental Psychology: Human Perception and Performance.
Logan, Miller, \& Strayer, 2011). Therefore, it seems likely that words constitute a unit of response selection in skilled typewriting.

However, there are reasons to think that letters (or keystrokes) are the responses that are selected. Keystrokes must be chosen and implemented in the correct order to type a word, and it is reasonable to consider the process that does that to be response selection. Moreover, many studies of response selection require pressing keys in response to letter stimuli (e.g., Eriksen \& Eriksen, 1974; Greenwald \& Shulman, 1973; Pashler, 1994b; Posner \& Snyder, 1975). These studies suggest that response selection occurs in the translation from a letter to a keypress. Hence, it is also possible that letters constitute the unit of response selection in skilled typewriting.

\section{Distinguishing Between Word and Letter Selection}

We distinguished these two alternatives with the PRP procedure (Pashler, 1994a; Welford, 1952). Skilled typists were asked to type a visually presented word (Task1) and respond vocally to a tone (Task2) concurrently or in a rapid succession. The stimuli for the two tasks (S1 and S2) were presented with a variable interval between them (stimulus onset asynchrony, or SOA). In most PRP tasks the response to S2 (R2) is delayed when SOA is short, whereas response to $\mathrm{S} 1$ (R1) is little influenced by SOA. This delay of R2 is known as the PRP effect. We focused on the PRP effect, asking how R2 queuing was affected by variables associated with typing in Task1.

The PRP effect is widely used as an operational definition of response selection. It is often explained in terms of a central bottleneck model that states that response selection can only operate on a single task at a time. The model generates a set of predictions about the outcomes of the PRP procedure, which can be used to identify the cognitive locus at which an experimental factor has its effect (e.g., McCann \& Johnston, 1992; Pashler, 1994a; Pashler \& Johnston, 1989; Van Selst, Ruthruff, \& Johnston, 1999). The most important prediction for our purposes is this (see Figure 2): a manipulation that prolongs the duration of prebottleneck or bottleneck process in Task1 increases the time that Task2 must wait for the bottleneck, increasing RT2 at short SOAs (Figure $2 \mathrm{a}$ vs. Figure $2 \mathrm{~b}$ ). In contrast, a manipulation that prolongs the duration of a postbottleneck process in Task1 does not affect RT2 (Figure 2a vs. Figure 2c). We took advantage of this wellestablished theory to examine whether response selection selects a word or a keystroke in skilled typewriting, or, in terms of the two-loop theory of typewriting (Logan \& Crump, 2011), whether the bottleneck is in the outer loop or the inner loop. ${ }^{1}$

In the present experiments, a critical manipulation was the length of words (or strings) to be typed, which ranged from three to five letters.

\footnotetext{
${ }^{1}$ Throughout the present article, we use the term "response selection" to refer to a bottleneck in performance in a way that is neutral with respect to the actual function. The term is often used to refer to a process of translating stimulus code to response code (e.g., McCann \& Johnston, 1992), but some researchers disagree (e.g., Hommel, 1998; Logan \& Gordon, 2001) and there is evidence of parallel stimulus-to-response translation in typing (Crump \& Logan, 2010a; Logan et al., 2011). There is also evidence that implies that the bottleneck may be a process that is closely related to response initiation/execution (e.g., Logan \& Burkell, 1986; Ulrich et al., 2006). We defer discussion of this issue until the General Discussion.
} 


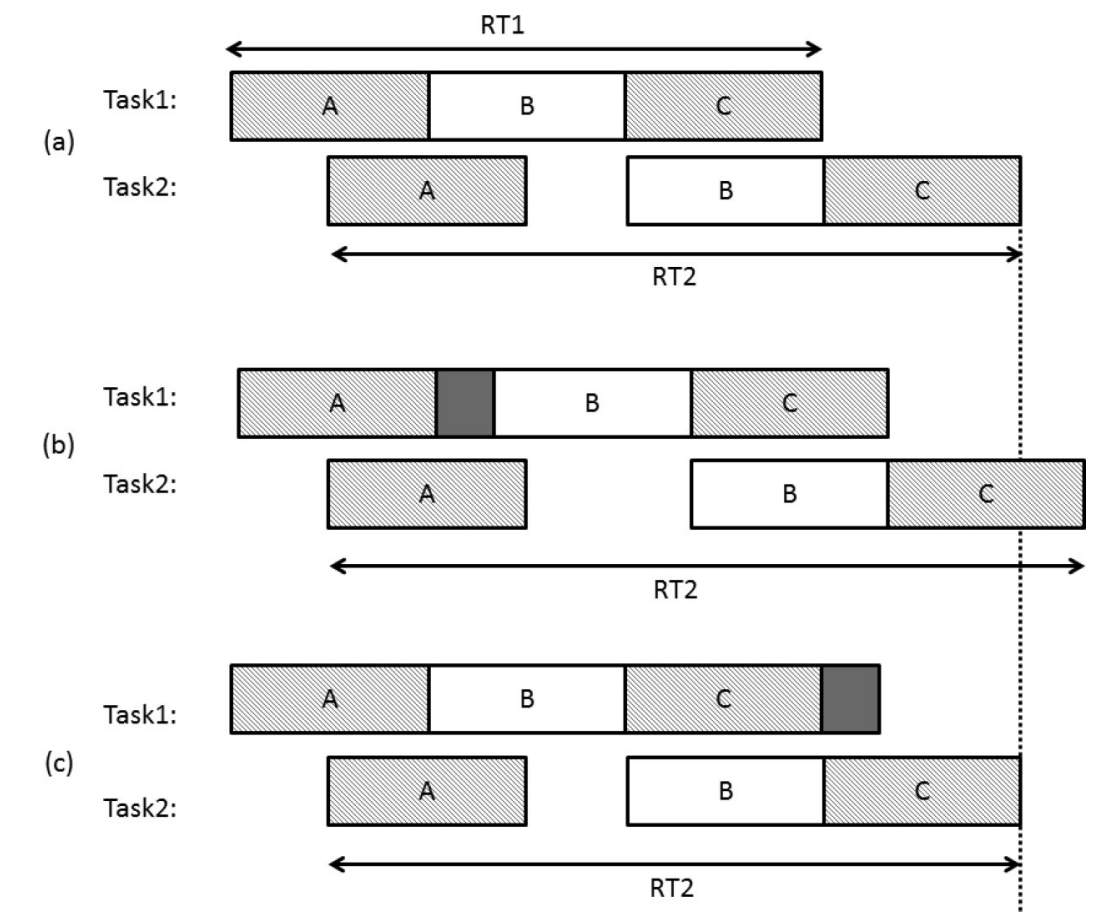

Figure 2. Illustrations of the effects of Task1 variables on RT2 (A = pre-bottleneck process; $\mathrm{B}=$ bottleneck process; $\mathrm{C}=$ post-bottleneck process; shaded areas are non-bottleneck processes). Gray solid areas represent the effect of Task1 variable on the pre-bottleneck or bottleneck process (b) or on the post-bottleneck process (c).

If response selection selects words (if the bottleneck is in the outer loop), then each trial requires selection of one response (a single word), so the PRP effect should be the same for all string lengths. By contrast, if response selection selects letters (if the bottleneck is in the inner loop), then each trial requires selection of as many responses as the number of letters in a word, so the PRP effect should increase with string length. Therefore, the locus of the bottleneck-in the outer loop or the inner loop — can be discovered by looking at the influence of string length on the PRP effect.

Alternatively, it is also possible that there is no PRP effect in the present task because subjects have had extensive training in typewriting. Indeed, Shaffer (1975b) demonstrated that professional typists could transcribe texts while shadowing spoken speech without much dual-task interference. However, Shaffer studied continuous typing and continuous tasks can be performed without dual-task interference by choosing an appropriate scheduling of the tasks (e.g., Broadbent, 1982; Pashler, 1994a; Van Selst et al., 1999; also see Byrne \& Anderson, 2001; Meyer \& Kieras, 1997; Ruthruff et al., 2003), so Shaffer's results might not mean there is no bottleneck in typing performance. The PRP procedure schedules the tasks independent of the subject and therefore disables the subject's scheduling strategies that might reduce dual-task interference. We conducted four experiments to address these issues.

\section{Predicted Effects of the String-Length Manipulation}

When the typing task is performed as Task 1 of the PRP procedure, there are several possible patterns of outcomes that could be obtained depending on the nature of typing performance. First, there may be no bottleneck in typing (see Figure 3a), so no PRP effect may be obtained. Second, the bottleneck in typing may be used to select words (i.e., the bottleneck is in the outer loop; see Figure 3b), so there would be a PRP effect but the effect would be independent of the number of letters that need to be typed. Third, the bottleneck in typing may be used to select letters (i.e., the bottleneck is in the inner loop). In this case, the PRP effect should be larger the more letters there are in a word, but the amount of the increase would depend on whether or not typing can be interrupted.

Logan (1982) found that typists could interrupt typing in a stop-signal experiment that required them to stop typing altogether when a tone occurred. However, the stop signal task is different from the present dual task in that it did not require typists to resume typing after they interrupted it. It is possible that typists may prefer to finish typing before beginning the second task in the PRP procedure. Whether they do so is an empirical question, which we addressed in these experiments. Predictions for interruptible and uninterruptible typing are derived formally in the Appendix. We present the results of the derivations here to illustrate the predictions.

If typing is uninterruptible, so that typists process all letters in a word before Task2 accesses the bottleneck (see Figure 3c-1), RT2 should increase by IKSI or a value close to it for each additional letter in S1. Formally, the expected difference in queuing time $\Delta Q T_{\text {unint }}$ between words with $n$ and $n+1$ letters is

$$
E\left[\Delta Q T_{\text {unint }}\right]=\sum_{i=1}^{n+1} p_{i} \cdot E\left[I K S I_{n+1}\right]-p_{n+1} \cdot E\left[d_{n+1}\right],
$$

where $p_{i}$ is the probability that Task 2 prebottleneck processes finish while the $i$ th keystroke occupies the bottleneck, $E\left[I K S I_{n+1}\right]$ 
(a) No bottleneck

\begin{tabular}{|c|c|c|c|}
\hline $\begin{array}{l}\text { Outer loop } \\
\text { (word) }\end{array}$ & $\begin{array}{l}\text { Inner loop } \\
\text { (letter) }\end{array}$ & $\begin{array}{l}\text { Inner loop } \\
\text { (letter) }\end{array}$ & $\begin{array}{l}\text { Inner loop } \\
\text { (letter) }\end{array}$ \\
\hline A & B & C & \\
\hline
\end{tabular}

(b) Select a word

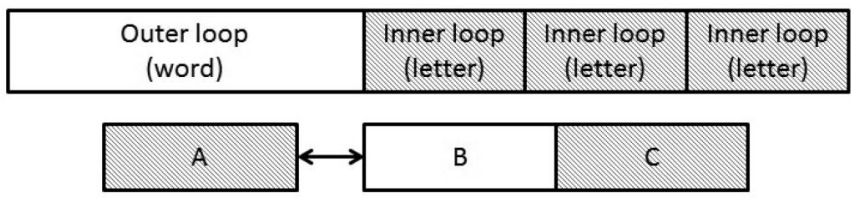

(c-1) Select a letter : Uninterruptible

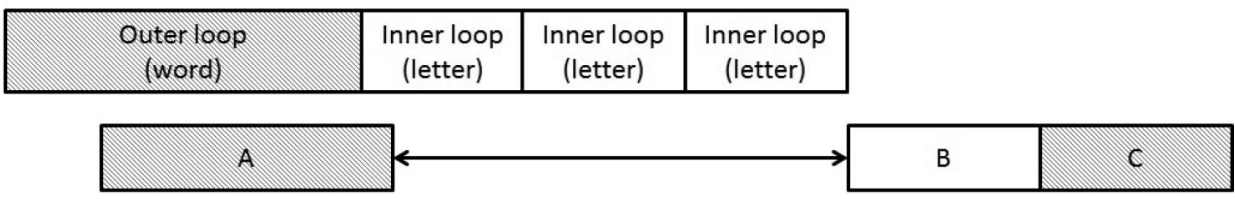

(c-2) Select a letter : Interruptible

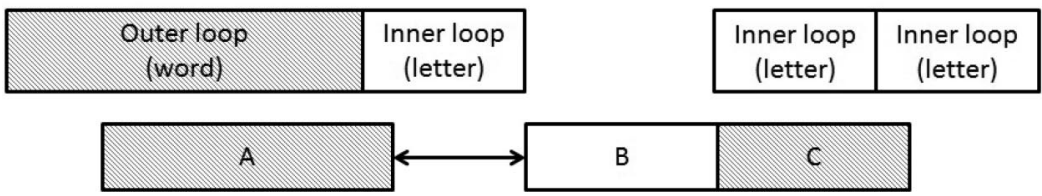

Figure 3. Illustrations of typing models in the PRP procedure $(\mathrm{A}=$ Task2 pre-bottleneck; $\mathrm{B}=$ Task2 bottleneck; $\mathrm{C}=$ Task2 post-bottleneck; shaded areas are non-bottleneck processes).

is the mean interkeystroke interval for the $(n+1)$ th keystroke, and $d_{n+1}$ is the portion of Task 2 prebottleneck processes that overlaps the bottleneck process of the last keystroke of the $n+1$ letter word. Note that if Task 2 prebottleneck processes always finish between the first keystroke and the last keystroke of the $n$ letter word (i.e., $\sum_{i=1}^{n} p_{i}=1$ ), then $\Delta Q T_{\text {unint }}$ is exactly equal to IKSI. If these values are greater than zero, $\Delta Q T_{\text {unint }}$ would be slightly smaller than IKSI. ${ }^{2}$

If typing is interruptible, so that typists can stop typing after executing any keystroke, perform Task 2 response selection, and resume typing, then the expected difference in queuing time $\Delta Q T_{\text {int }}$ between words with $n$ and $n+1$ letters is

$$
E\left[\Delta Q T_{i n t}\right]=p_{n+1} \cdot E\left[I K S I_{n+1}-d_{n+1}\right],
$$

All quantities in the equation are associated with the interval between the last keystrokes of the shorter word and the last keystroke of the longer word. This follows because queuing time will be the same regardless of string length if Task2 prebottleneck processes finish before the last keystroke of the shorter word. In those cases, Task 2 response selection can occur as soon as the current keystroke is finished. Queuing time will only differ between string lengths if Task2 prebottleneck processes finish after the last letter of the shorter word and before the last letter of the longer word. Thus, if Task2 prebottleneck processes always complete before the $(n+1)$ th keystroke, (i.e., $\left.p_{n+1}=0\right)$, then $\Delta Q T_{i n t}$ is exactly equal to zero. If Task 2 prebottleneck processes some- times finish when the $(n+1)$ th keystroke occupies the bottleneck (i.e., $p_{n+1}>0$ ), $\Delta Q T_{\text {int }}$ is greater than zero.

Uninterruptible and interruptible typing can also be distinguished by noting where $\mathrm{R} 2$ occurs with respect to the word. If typing is uninterruptible, R2 should occur before the first keystroke or after the last keystroke, and never between the first and last keystrokes. On the other hand, if typing is interruptible, R2 could occur between any pair of successive keystrokes. These predictions can be examined directly from the data by looking at the position of $\mathrm{R} 2$ relative to the keystrokes.

Finally, typing performance may be a mixture of uninterruptible and interruptible cases. For instance, typing may be interruptible for more skilled typists but uninterruptible for less skilled typists, or vice versa. Typing may also be interruptible for some words but uninterruptible for other words (e.g., high vs. low frequency words), or it may be uninterruptible for some sequences of keystrokes but interruptible for other sequences. Then, the effect of string length will be intermediate between zero and IKSI, and the mixture proportion can be obtained by

\footnotetext{
${ }^{2} E\left[d_{n+1}\right]$ can be expected to be small at short SOAs because only the long tail of the Task2 pre-bottleneck latency distribution would overlap with the last keystroke of longer words, and the tail would be skewed positively, which makes the mean of this truncated latency distribution (i.e., $\left.E\left[d_{n+1}\right]\right)$ small. Also, as can be seen in Equation 1, the term is weighted by $p_{\mathrm{n}+1}$, which would also be small at short SOAs.
} 
dividing the observed increase in RT2 at short SOAs by mean IKSI; that is,

$$
\begin{aligned}
E[\Delta Q T] & =P \cdot E\left[\Delta Q T_{\text {unint }}\right]+(1-P) \cdot E\left[\Delta Q T_{\text {int }}\right] \\
& =P \cdot E[I K S I],
\end{aligned}
$$

so $P=E[\Delta Q T] / E[I K S I]$ is the proportion of the uninterruptible case in the data. ${ }^{3}$

\section{Experiment 1}

Does response selection select words or letters in skilled typewriting? In Experiment 1, Task1 required skilled typists to type a single word on each trial, whereas Task2 required typists to respond vocally to tones by saying " $H I G H$ " or " $L O W$ " to indicate the pitch of tones. If typing involves a response selection bottleneck, the PRP effect should be obtained in Task2. To assess the locus of the bottleneck, we varied the length of words (three, four, or five letters) across trials. If typing involves a response selection bottleneck in the outer loop (if response selection selects single words; see Figure 3b), the string-length manipulation should have no effect on RT2, producing the same magnitude of the PRP effect across trials with different string lengths. If typing involves a response selection bottleneck in the inner loop (if response selection selects individual letters; see Figure 3c), the string-length manipulation should have a strong effect; RT2 should be delayed more for longer words and produce a larger PRP effect.

If word length affects RT2, we can ask whether typing is interruptible or uninterruptible (see Appendix). If typing is uninterruptible, then RT2 at short SOAs should increase by IKSI for each additional letter (see Equation 1 and Figure 3c-1). If typing is interruptible, then the increase in RT2 with word length should not be as large as IKSI and possibly equal to zero (see Equation 2 and Figure $3 \mathrm{c}-2$ ). If typing is uninterruptible sometimes and interruptible the other times, then the effect of the string length would be intermediate; that is, larger than zero but smaller than IKSI. The mixture proportion can be obtained by dividing IKSI by the amount of increase in the PRP effect per letter (see Equation 3). To determine which factors affect the mixture proportion, we assess influences of skill level of typists, word frequency, and word difficulty on the string length effect on RT2.

\section{Method}

\section{Subjects}

Sixteen touch-typists were recruited from the Vanderbilt University Community (10 females; mean age $=23.38, S D=4.53$ ). They received $\$ 12$ for a $1-\mathrm{hr}$ experimental session. All typists reported having normal or corrected-to-normal visual acuity and normal hearing. A typing test was administered to each typist (see Logan \& Zbrodoff, 1998). Their mean typing speed was 80.76 words per minute (WPM; $S D=15.77)$ and their accuracy was $95.39 \%$ ( $S D=1.96)$. On average, these typists had 4.07 months of formal training in typing (one or two semester courses; $S D=2.40$ ) and 12.66 years of typing experience $(S D=3.62)$. They reported spending 4.53 hours per day by typing $(S D=3.08$ ).

\section{Apparatus and Stimuli}

The apparatus consisted of a personal computer connected to a 19-in color CRT monitor. Stimuli were words presented at the center of screen, in 18-pt Courier New font, and sinusoidal tones. The words were printed in black and presented against a white background in upper case. ${ }^{4}$ The words consisted of three, four, or five letters (see below for more details). Responses to the words were registered by typing on the QWERTY keyboard. The tones were high- and low-pitched (800 and $400 \mathrm{~Hz}$ ) and presented through headphones. The intensity was adjusted for each subject at a comfortable level, with an approximate range of 45-55 dB. Responses to the tones were registered by speaking into a microphone placed in front of the computer monitor, and the experimenter pressed keys on a response box to record the identity of the vocal responses. The microphone was also connected to the response box, which registered voice onset time.

Word lists. The word lists were constructed as follows: The MRC Psycholinguistic Database ${ }^{5}$ was used to generate the initial sets of three-letter words $(n=266)$, four-letter words $(n=1,365)$, and five-letter words $(n=792)$, and the Corpus of Contemporary American English ${ }^{6}$ was used to obtain the word frequencies of these words per million. From the three-letter word list, 200 words (nouns, verbs, and adjectives) were selected arbitrarily. Then, 200 words were chosen from each of the four- and five-letter word lists, such that the distributions of word frequencies for the selected words were equivalent among the three lists. The equivalence of the frequency distributions was ensured in the following manner: First, 200 words were randomly selected from each of the four- and five-letter word lists and were submitted to a two-sample Kolmogorov-Smirnov (K-S) test to compare the frequency distribution of each list with that of the three-letter word list. If the null hypothesis was rejected, a new set of 200 random samples were selected from the initial word list, and the K-S test was administered again. This procedure was repeated until the null hypothesis was retained. Finally, the word frequencies of the three lists were submitted to ANOVA, which suggested no significant difference among the lists; $F(2,597)=0.65, M S E=114,524$. Mean word frequencies were $140.72,148.08$, and $111.58(S E \mathrm{~s}=24.25,24.25$,

\footnotetext{
${ }^{3}$ This assumes that Task2 pre-bottleneck processes always complete between the first keystroke and the last keystroke of the shorter word; i.e. $p_{i}=0$ for $i=0$ or $i>n$. If the assumption does not hold, $P$ is given by the following:

$$
p=\frac{1}{\sum_{i=1}^{n} p_{i}}\left(\frac{E[\Delta Q T]}{E[I K S I]}\right)-\frac{p_{n+1}}{\sum_{i=1}^{n} p_{i}}\left(1-\frac{E\left[d_{n+1}\right]}{E[I K S I]}\right),
$$

so Equation 3 likely overestimates the actual proportion of the uninterruptible case.

${ }^{4}$ A reviewer pointed out the possibility that presentation of word stimuli in upper case might alter the nature of typing words because it differs from normal copy typing in which letters are usually presented in lower case (except for the first letter). We do not think that this has a practical consequence from results of previous typing studies. For instance, Crump and Logan (2010a) presented words in upper case as a prime and found evidence indicating parallel activation of keystrokes, which was absent when they presented nonwords in upper case, suggesting a unique property of typing words. Such findings imply that uppercase presentation does not alter the processes underlying typing words.

${ }^{5}$ http://www.psy.uwa.edu.au/mrcdatabase/-uwa_mrc.htm

${ }^{6} \mathrm{http}: / / \mathrm{www}$.americancorpus.org/
} 
and 18.80), for the three-letter, four-letter, and five-letter word lists, respectively.

\section{Procedure}

The experiment was conducted individually under normal fluorescent lighting. Subjects were seated in front of the computer at an unrestricted viewing distance of $55 \mathrm{~cm}$. The experimenter also sat in the experimental room to register subjects' vocal responses. Subjects read on-screen instructions for the task.

The experiment consisted of a PRP procedure for which Task1 was a typing task and Task2 was a tone discrimination task. Each subject performed one block of six practice trials for Task1 (two trials for each string length), one block of six practice trials for Task2, and one block of 24 practice trials for a dual-task condition for which both tasks were performed in each trial. Then, they performed 480 test trials in the dual-task condition. Subjects were given a brief rest period at every 120 test trials. For each subject, 170 words were randomly selected from each list of the three string lengths (10 words for practice trials and 160 words for test trials). A session took less than an hour for each subject.

Each trial started with a fixation cross at the center of screen that appeared for $500 \mathrm{~ms}$. A word stimulus (S1) replaced the cross, and subjects were asked to start typing the word as quickly and as accurately as they could. Trials with three-, four-, and five-letter words were intermixed and presented randomly. As subjects pressed a key, the corresponding letter appeared in a lower case below the to-be-typed letter of the word stimulus at a vertical distance of $5 \mathrm{~cm}$. With a variable SOA $(100,300,900$, or 1800 $\mathrm{ms}$ ), a tone (S2) was presented binaurally through headphones. The tone was either high- or low-pitched, and subjects said "HIGH" or "LOW" according to the pitch. When vocal response triggered voice key, the experimenter pressed a key on a response box to register the response. If the response was ambiguous, or if the microphone appeared to have registered a noise before subjects uttered the actual response, the experimenter recorded the trial as ambiguous. Such trials were excluded from the subsequent analysis.

Subjects were instructed not to stop typing to respond to tones or delay the vocal responses to complete typing. They were asked to respond to both stimuli as quickly and as accurately as possible and were encouraged to perform two task concurrently if needed. A trial ended when subjects finished typing the word and made a vocal response or when five seconds had elapsed since the onset of the word. As feedback to the responses, the messages "CORRECT" and "WRONG" were displayed for correct and error responses, respectively. The feedback for Task1 appeared $2.5 \mathrm{~cm}$ above the screen center, and that for Task 2 appeared $2.5 \mathrm{~cm}$ below the screen center. Both messages were printed in blue, and they remained on the screen for $1,000 \mathrm{~ms}$, followed by the fixation cross, which signaled the beginning of the next trial.

Response time for Task1 (RT1) was the interval between word onset and the first keypress, and response time for Task2 (RT2) was the interval between tone onset and onset of vocal response. Interkeystroke interval (IKSI) was the interval between two successive keystrokes for the typing task.

\section{Results}

Trials for which RT1 or RT2 were shorter than $200 \mathrm{~ms}$ were considered anticipations and were not included in the analysis $(<0.58 \%)$. Mean RT1, RT2, and IKSI for correct trials were computed for each subject as a function of String Length (three, four, and five letters) and SOA (100, 300, 900, and $1800 \mathrm{~ms})$. For Task1, correct trials were those for which the whole word was typed correctly. Percentage errors for Task1 (PE1) and Task2 (PE2) were analyzed in the same design. RT1 and RT2 are summarized in Figure 4, and IKSI, PE1, and PE2 are summarized in Tables 1, 2, and 3, respectively. Each dependent variable was submitted to 3 (String Length: three, four, and five letters) $\times 4$ (SOA: 100, 300, 900, $1800 \mathrm{~ms}$ ) ANOVAs, and the results are summarized in Table 4. The only significant effect in PE was a main effect of String Length in PE1 (see Table 2), reflecting that longer words produced more errors, most likely because there were more opportunities to make errors. Hence, we focus on RT and IKSI.

\section{Does String Length Affect the PRP Effect?}

The most important finding is the PRP effect in RT2 (see Figure 4): RT2 was longer for the 100-ms SOA than for 1800-ms SOA by $200 \mathrm{~ms}$ on average. RT2 also depended on String Length, and String Length interacted with SOA. The difference in RT2 between the 100- and 1800-ms SOAs was $150 \mathrm{~ms}$ for three-letter words, $189 \mathrm{~ms}$ for four-letter words, and $214 \mathrm{~ms}$ for five-letter words. Thus, the PRP effect increased as the number of letters to be typed increased. The increase of RT2 was $34 \mathrm{~ms} / \mathrm{keystroke}$ at the 100-ms SOA, $45 \mathrm{~ms} /$ keystroke at the 300-ms SOA, $22 \mathrm{~ms} /$ keystroke at the 900-ms SOA, and $2 \mathrm{~ms} /$ keystroke at the $1800-\mathrm{ms}$ SOA. Although RT1 also increased by $10 \mathrm{~ms} /$ keystroke as string length increased (RT1s $=604,613$, and $625 \mathrm{~ms}$, for three-, four-, and five-letter words, respectively), the increase was much smaller than that obtained for RT2 at the shorter SOAs. Overall, these outcomes indicate that selection of a keystroke constitutes a bottleneck in typewriting.

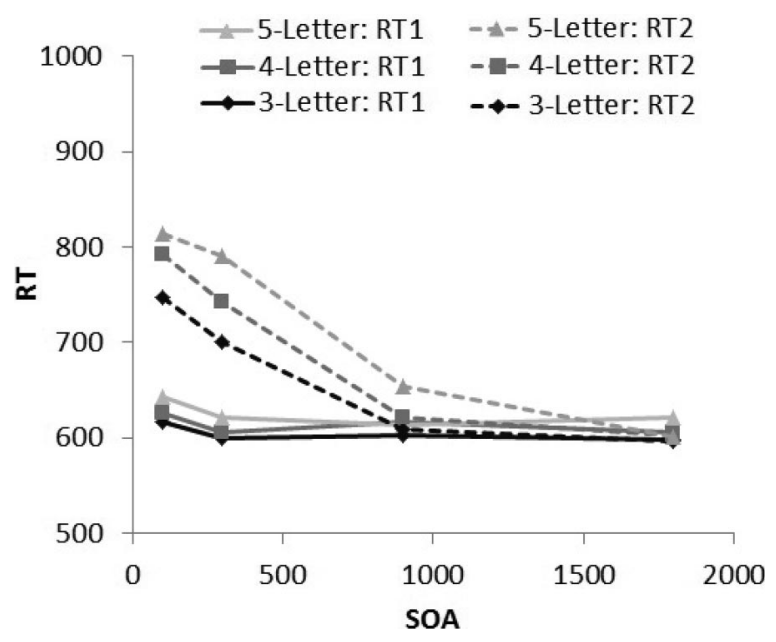

Figure 4. Response times for Typing Task1 (RT1) and Tone Task2 (RT2) in Experiment 1 ( $\mathrm{SOA}=$ stimulus onset asynchrony). 
Table 1

Interkeystroke Intervals as a Function of Stimulus Onset Asynchrony and String Length for Word and Nonword Trials

\begin{tabular}{llllllll}
\hline & \multicolumn{3}{c}{ Word trials } & & \multicolumn{3}{c}{ Nonword trials } \\
\cline { 2 - 3 } \cline { 6 - 7 } SOA & 3-letter & 4-letter & 5-letter & & 3-letter & 4-letter & 5-letter \\
\hline Experiment 1 & & & & & & & \\
$\quad 100$ & 137 & 138 & 144 & & - & - & - \\
300 & 138 & 135 & 140 & & - & - & - \\
900 & 132 & 128 & 136 & & - & - & - \\
1800 & 135 & 130 & 134 & & - & - & - \\
Experiment 2 & & & & & & \\
100 & 133 & 139 & 140 & & 170 & 185 & 200 \\
300 & 134 & 134 & 138 & & 173 & 184 & 196 \\
1200 & 135 & 131 & 139 & & 169 & 181 & 194 \\
Experiment 3 & & & & & & \\
100 & 114 & 127 & 130 & & 123 & 137 & 158 \\
300 & 118 & 130 & 133 & & 120 & 139 & 162 \\
1200 & 122 & 125 & 133 & & 126 & 148 & 165 \\
Experiment 4 & & & & & & \\
100 & 130 & 127 & 133 & & 156 & 174 & 192 \\
300 & 127 & 127 & 129 & & 160 & 173 & 189 \\
1200 & 122 & 123 & 128 & & 152 & 164 & 175 \\
\hline
\end{tabular}

Note. $\quad \mathrm{SOA}=$ Stimulus Onset Asynchrony.

There was a PRP-like effect in RT1 (see Figure 4): RT1 was longer at the $100-\mathrm{ms} \operatorname{SOA}(M=628 \mathrm{~ms})$ than at longer SOAs (Ms $=609,611,608 \mathrm{~ms}$, for the 300-, 900-, and 1800-ms SOAs). This outcome could be either attributable to task reversal (i.e., Task 2 occupies the bottleneck first, so Task 1 is queued; Miller, Ulrich, \& Rolke, 2009) or capacity sharing (i.e., the rate of Task1 processing decreases because less than the maximum capacity is allocated to Task1; Navon \& Miller, 2002; Tombu \& Jolicœur, 2003). If it was attributable to task reversal, removing trials for which RT1 was longer than RT2 would reduce or eliminate the effect, whereas the removal would have no consequence if it was attributable to capacity sharing. The data supported task reversal; the PRP-like effect in RT1 disappeared when trials in which R2 occurred before the first keystroke, $F(3.45)<1, M S E=1,102$, $\eta_{p}^{2}=.029$ (RT1 were 609, 603, 611, $608 \mathrm{~ms}$, for the 100-, 300-, 900-, and 1800-ms SOAs). Nevertheless, the removal did not affect statistical outcomes of RT2 analyses.

\section{Is Typing Uninterruptible or Interruptible?}

The amount of increase in the PRP effect per letter provides important implications as to the nature of typing performance (see Equation 1-3 and the Appendix). The PRP effect would increase by IKSI per letter or close to that value if typing is uninterruptible, and the effect would not increase that much if typing is interruptible. Mean IKSI was $136 \mathrm{~ms}$ (see Table 1). The string-length effect was statistically significant in IKSI, but the pattern was not systematic (IKSIs were 135,133, and 138 ms, for three-, four-, and five-letter words). This effect was not replicated in the subsequent experiments (also see Shaffer \& Hardwick, 1969). The observed increase of RT2 at the 100-ms SOA was $34 \mathrm{~ms} /$ letter on average, which was significantly larger than zero (one-sample $t$ test with the null hypothesis " $E[\Delta Q T]=0 ": t(15)=4.30, S E=8.40, p<.001)$, providing no support for the outer-loop locus of the PRP effect. The increase was also significantly smaller than IKSI (pairedsamples $t$ test with the null hypothesis " $E[\Delta Q T]=E[I K S I]$ ": $t(15)=11.21, S E=8.86, p<.001)$, which is inconsistent with

Table 2

Percentage Errors for Task1

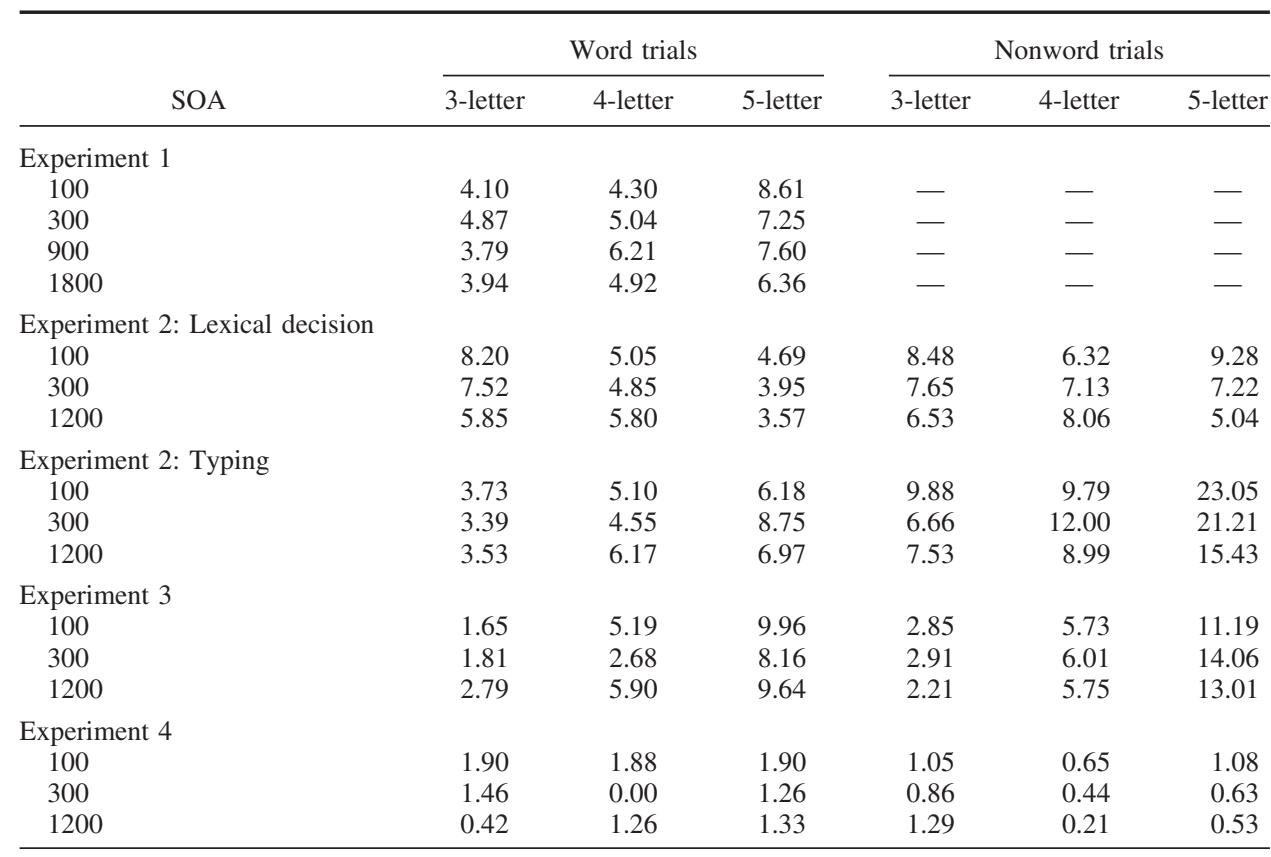

Note. $\quad$ SOA $=$ Stimulus Onset Asynchrony. 
Table 3

Percentage Errors for Task2

\begin{tabular}{|c|c|c|c|c|c|c|}
\hline \multirow[b]{2}{*}{ SOA } & \multicolumn{3}{|c|}{ Word trials } & \multicolumn{3}{|c|}{ Nonword trials } \\
\hline & 3-letter & 4-letter & 5-letter & 3-letter & 4-letter & 5-letter \\
\hline \multicolumn{7}{|l|}{ Experiment 1} \\
\hline 100 & 2.37 & 1.76 & 2.71 & - & - & - \\
\hline 300 & 1.57 & 2.51 & 1.81 & - & - & - \\
\hline 900 & 1.89 & 1.27 & 1.11 & - & - & - \\
\hline 1800 & 1.74 & 0.96 & 0.79 & - & - & - \\
\hline \multicolumn{7}{|c|}{ Experiment 2: Lexical decision } \\
\hline 100 & 1.84 & 1.30 & 0.97 & 4.07 & 2.23 & 3.41 \\
\hline 300 & 1.31 & 1.48 & 1.52 & 1.66 & 1.89 & 1.49 \\
\hline 1200 & 1.33 & 1.33 & 0.56 & 0.00 & 1.14 & 1.12 \\
\hline \multicolumn{7}{|c|}{ Experiment 2: Typing } \\
\hline 100 & 1.13 & 0.55 & 0.94 & 0.94 & 2.95 & 2.10 \\
\hline 300 & 1.35 & 0.56 & 1.48 & 2.98 & 3.06 & 2.88 \\
\hline 1200 & 0.37 & 0.94 & 0.75 & 0.57 & 0.83 & 3.57 \\
\hline \multicolumn{7}{|l|}{ Experiment 3} \\
\hline 100 & 1.26 & 3.17 & 1.31 & 1.61 & 1.99 & 2.45 \\
\hline 300 & 1.27 & 1.21 & 1.76 & 0.60 & 1.42 & 2.07 \\
\hline 1200 & 0.92 & 0.75 & 1.37 & 0.70 & 0.24 & 1.88 \\
\hline \multicolumn{7}{|l|}{ Experiment 4} \\
\hline 100 & 2.95 & 5.25 & 7.50 & 7.65 & 9.62 & 13.16 \\
\hline 300 & 3.15 & 5.68 & 8.27 & 8.19 & 9.24 & 13.48 \\
\hline 1200 & 4.59 & 1.50 & 5.26 & 8.32 & 8.03 & 9.06 \\
\hline
\end{tabular}

Note. $\quad \mathrm{SOA}=$ Stimulus Onset Asynchrony.

the uninterruptible case of the inner-loop locus. Both results are consistent with the interruptible case.

To determine whether typing was uninterruptible or interruptible, we computed the proportions of trials for which R2 occurred before the respective keystrokes for each combination

Table 4

ANOVA Results for Experiment 1

\begin{tabular}{lccrc}
\hline \multicolumn{1}{c}{ Factor } & $d f$ & $F$ & $M S E$ & $\eta_{p}^{2}$ \\
\hline RT1 & & & & \\
$\quad$ String length (SL) & 2,30 & $19.57^{*}$ & 349 & .566 \\
$\quad$ Stimulus onset asynchrony & & & & \\
$\quad$ (SOA) & 3,45 & $4.41^{*}$ & 961 & .227 \\
$\quad$ SL $\times$ SOA & 6,90 & 1.88 & 233 & .111 \\
IKSI & & & & \\
SL & 2,30 & $8.4^{*}$ & 56 & .357 \\
SOA & 3,45 & $7.60^{*}$ & 91 & .336 \\
SL $\times$ SOA & 6,90 & 1.34 & 37 & .082 \\
RT2 & & & & \\
SL & 2,30 & $17.85^{*}$ & 2390 & .633 \\
SOA & 3,45 & $25.90^{*}$ & 14672 & .633 \\
SL $\times$ SOA & 6,90 & $6.37^{*}$ & 916 & .298 \\
PE1 & & & & \\
SL & 2,30 & $14.34^{*}$ & 12.72 & .489 \\
SOA & 3,45 & $<1$ & 21.62 & .018 \\
SL $\times$ SOA & 6,90 & 1.06 & 10.36 & .066 \\
PE2 & & & & \\
SL & 2,30 & $<1$ & 2.55 & .042 \\
SOA & 3,45 & 1.90 & 6.49 & .113 \\
SL $\times$ SOA & 6,90 & 1.03 & 4.10 & .064 \\
\hline
\end{tabular}

Note. $\mathrm{RT}=$ response time; IKSI $=$ interkeystroke interval; $\mathrm{PE}=$ percentage error.

${ }^{*} p<.01$ of String Length and SOA for each subject. Means across subjects are plotted in Figure 5. If typing is uninterruptible, R2 should have occurred before the first keystroke or after the last keystroke, but the data suggest that R2 could be inserted between any pair of successive keystrokes. For five-letter words, for instance, R2 occurred before the first keystroke in $13 \%$ of trials, between the first and second keystrokes in $21 \%$ of trials, between the second and third keystrokes in $25 \%$ of trials, between third and fourth keystrokes in $19 \%$ of trials, between the fourth and fifth keystrokes in $11 \%$ of trials, and after the fifth keystroke in $10 \%$ of trials. There were similar percentages for three-letter words $(M \mathrm{~s}=11 \%, 26 \%, 28 \%$, and $35 \%)$ and for four-letter words $(M \mathrm{~s}=12 \%, 22 \%, 25 \%, 20 \%$, and $22 \%)$. These data are inconsistent with the uninterruptible model but are consistent with the interruptible model.

\section{Is Typing a Mixture of Interruptible and Uninterruptible Cases?}

If typing performance is a mixture of the interruptible and uninterruptible cases, the string length effect should be somewhere between zero and IKSI and R2 could be inserted in any pair of successive keystrokes. Applying Equation 3, the ratio of the increase in RT2 at the 100-ms SOA to IKSI was 0.26, suggesting that typing may be uninterruptible $26 \%$ of the time (i.e., for $26 \%$ of subjects, words, digraphs, etc.).

To assess the influence of skill level, we split the typists into two groups with equal number $(N \mathrm{~s}=8)$ according to their typing speed during the typing test administered at the beginning of the session ( $M \mathrm{~s}=67.76$ vs. 93.77 WPM). The mixture proportions (Equation 3) were computed for each typist and submitted to an independent $t$ test. The proportions were similar 

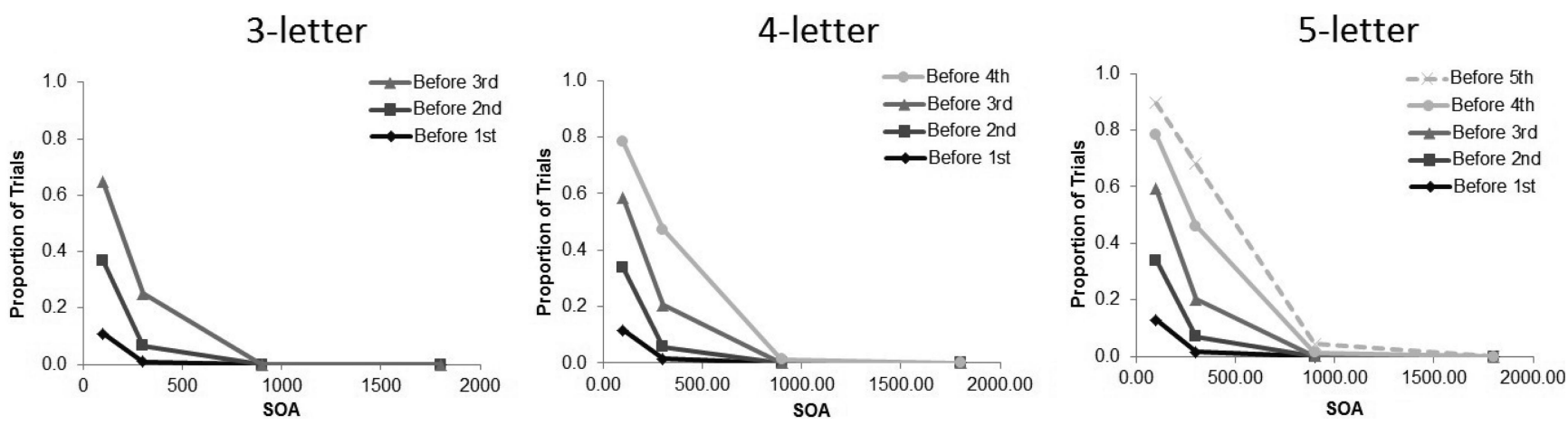

Figure 5. Proportions of trials for which R2 preceded the respective keystrokes for three-, four-, and five-letter words in Experiment 1 ( $\mathrm{SOA}=$ stimulus onset asynchrony).

between the two groups $(0.24$ for more skilled typists and 0.24 for less skilled typists), $t(14)=.004, S E=.10$. To assess the influence of word frequency, we split all trials at the median frequency for each combination of SOA and String Length and computed the mixture proportions for each typist and submitted to a paired $t$ test. The mixture proportions for high and low frequency words were 0.27 and 0.18 , which did not differ significantly, $t(15)=1.06, S E=.10$. Finally, to examine the influence of word difficulty, we split trials according to the difficulty of digraphs involved in typing the respective words ${ }^{7}$ and computed the mixture proportions. The mixture proportions were 0.26 for less difficult words and 0.20 for more difficult words, but they did not differ significantly either, $t(15)=.54$, $S E=.11$. Therefore, the mixture proportions were not influenced by skill level, word frequency, or word difficulty. Although it is still possible that there exist factors other than the ones considered here that determine whether or not typing is interruptible, further studies need to be designed specifically to identify and examine such sources of mixtures.

\section{Discussion}

The present experiment established that a bottleneck exists in skilled typing. Furthermore, RT2 increased with the number of letters in words to be typed in Task1. The results suggest that response selection in skilled typing selects letters or keystrokes, so the bottleneck is in the inner loop rather than the outer loop. The data suggest that response selection in typing is interruptible and can be interrupted after any keystroke has been selected, corroborating Logan (1982) in a dual-task procedure. Hence, we conclude that Task 2 response selection is queued until the selection of the current keystroke is completed. In Experiments 2 and 3, we sought convergent evidence supporting this conclusion.

\section{Experiment 2}

The second experiment was designed to determine whether the effects in Experiment 1 were attributable to typing or to processing words. We compared the typing task from Experiment 1 with a lexical-decision task (i.e., judging whether S1 was a word or nonword), which requires encoding and lexical access like the outer-loop processing in the typing task. Thus, if the effects in Experiment 1 were attributable to the outer loop, similar effects of string length should be obtained when Task1 requires lexical decision. However, if the effects were attributable to the inner loop, no string length effects should be obtained in lexical decision because the task requires only a single response (indicating a word or nonword) regardless of string length. We were interested in word length effects in both RT1 and RT2. RT1 effects reflect outer loop processing as well as inner loop processing. Thus, we expected to see the same word length effects on RT1 for typing and lexical decision. The data from Experiment 1 suggest that the RT2 effects reflect inner loop processing, so we expected different word length effects on RT2 for typing and lexical decision.

In the present experiment, each subject participated in two experimental sessions. In one of the sessions, they performed a PRP procedure in which Task1 required lexical decision and Task2 required a tone discrimination task; in the other session, they performed a PRP procedure in which Task1 required typing a word or nonword and Task 2 required the same tone discrimination task. The nonword condition was required for the lexical-decision task (subjects had to discriminate words from nonwords) but it was also interesting as a typing task. Typists may select responses in nonwords one letter at a time (Crump \& Logan, 2010a), so we might see more interruption with nonwords than with words. Also, typing nonwords is typically slower than typing words, so the word length effect on RT2 at short SOAs might be larger for nonwords than for words.

\footnotetext{
${ }^{7}$ All digraphs were first classified into four categories; "double" (digraphs that are typed using the same keys), "same finger" (digraphs that are typed using the same finger but different keys), "same hand" (digraphs that are typed using the same hand but different fingers), and "different hands" (digraphs that are typed using different hands), and IKSI was computed $(M \mathrm{~s}=123,134,150$, and $188 \mathrm{~ms}$, for different hands, same hands, double, and same finger, respectively). The difficulty indices between 1 and 4 were assigned to the digraph categories according to IKSI ( 1 being the easiest), and the difficulty of typing each word was determined by summing the indices associated with the word. For instance, a trial with the word "ONCE" had the difficulty score of 2 (same hand) +1 (different hands) + $4($ same finger $)=7$.
} 


\section{Method}

\section{Participants}

A new group of 16 touch-typists were recruited from the same subject pool as in the preceding experiment, using the same criteria for subject selection (nine females; mean age $=22.25, S D=$ 2.89). Each typist participated in two experimental sessions. These sessions were administered on different days of the same week. They received $\$ 12$ for participating in each session. Mean typing speed was 81.09 WPM $(S D=18.09)$ and mean accuracy was $95.74 \%$ ( $S D=3.16$ ). They had 5.04 months of formal training in typing on average $(S D=3.96)$ and 11.38 years of typing experience $(S D=3.38)$. They reported spending 3.88 hours per day for typing $(S D=1.60)$.

\section{Apparatus, Stimuli, and Procedure}

The apparatus was the same as that used in Experiment 1. For one of the two experimental sessions, Task1 was a lexical-decision task, in which subjects pressed one of two keys ("z" or " ") to indicate whether $\mathrm{S} 1$ was a word or nonword. The assignment of the response keys to the response categories was counterbalanced across subjects. For the other session, Task1 was a typing task similar to the task used in Experiment 1. Half of the typists performed lexical decision in the first session and typing in the second session; the order was reversed for the other half.

For both the typing and lexical decision tasks, word lists were the same as those used in Experiment 1. Nonword lists were prepared by randomly scrambling the order of the letters in the words with the constraint that the first and last letters were not at the original locations. When the resulting items were common words or familiar acronyms, one of the letters in the item was arbitrarily chosen and replaced with another letter whose key was adjacent to the original key. Also, to reduce the probability of task order reversal (i.e., to encourage responding to S1 before S2), S1 was presented only for $500 \mathrm{~ms}$.

Task2 was the same tone discrimination task used in Experiment 1 , in which subjects uttered "HIGH" or "LOW" according to the tone pitch. Instead of the desktop microphone used in Experiment 1 , a headset microphone was used to register vocal responses. The stimuli for Task2 were those used in Experiment 1. In the present experiment, the experimenter did not sit in the experiment room. Instead, vocal responses were recorded on each trial, and the experimenter analyzed them after each session. The duration of each trial was fixed at 3,000 ms to record vocal responses. RT2 was also determined subsequently using the recorded voice data for each trial. Because of the uncertainty about vocal responses during the experiment, the trial-to-trial feedback was not given for Task2. Feedback to Task1 was provided in the same manner as in Experiment 1.

There were three SOAs (100, 300, and $1200 \mathrm{~ms})$ and three string lengths (three, four, and five letters) for each of word and nonword trials. The string lengths and SOAs were randomly intermixed in each block. Each session had six test blocks, consisting of 102 trials each.

\section{Results}

Trials were filtered in the same manner as in Experiment 1 $(<0.84 \%$ of the trials were discarded in the lexical decision session; $<1.74 \%$ in the typing session). We analyzed the data of the lexical decision session and the typing session separately. Mean RT1 and RT2 for correct trials are shown in Figure 6A for the lexical decision session and in Figure 6B for the typing session. IKSI for the typing session is shown in Table 1, and PE1 and PE2 for both sessions are shown in Tables 2 and 3, respectively. RT1, RT2, IKSI, PE1, and PE2 were submitted to 3 (SOA: 100, 300, $1200 \mathrm{~ms}) \times 3$ (String Length: 3, 4, 5 letters) $\times 2$ (Stimulus Type: word, nonword) ANOVAs. The results are shown in Table 5 for the lexical decision session and in Table 6 for the typing session.

\section{Lexical Decision Session}

For lexical decision (see Figure 6A), there was a large PRP effect; RT2 was $271 \mathrm{~ms}$ longer at the $100-\mathrm{ms}$ SOA than at the 1200-ms SOA. However, the PRP effect did not differ among the three string lengths: At the 100-ms SOAs, RT2 was similar for the three string lengths $(M \mathrm{~s}=860,873$, and $873 \mathrm{~ms})$. String length did not affect RT1 either: RT1 was 576, 586, and $580 \mathrm{~ms}$ for three-, four-, and five-letter words. This suggests that encoding, lexical access, and response selection in lexical decision are not affected by string length.

RT2 was longer for nonwords $(M=742 \mathrm{~ms})$ than for words ( $M=727 \mathrm{~ms})$, but RT1 did not differ between nonwords $(M=$ $580 \mathrm{~ms})$ and words $(M=581 \mathrm{~ms})$. RT1 was shorter at shorter SOAs $(M \mathrm{~s}=573,579$, and $590 \mathrm{~ms}$, for the 100-, 300-, and 1200-ms SOAs). This RT1 pattern suggests that subjects might have grouped responses, waiting for S2 to occur before responding to S1 in some trials (Ulrich \& Miller, 2008).

PE1 (see Table 2) was larger for shorter strings $(M \mathrm{~s}=7.34 \%$, $6.03 \%$, and $5.48 \%$, for three, four, and five letters). The effect seems to reflect the possibility that shorter nonwords tend to be more similar to words (e.g., Yap, Balota, Cortese, \& Watson, 2006). Finally, PE2 increased at shorter SOAs (see Table 3), yielding a PRP-like effect, and this effect was more apparent for nonwords $(M \mathrm{~s}=3.24 \%, 1.68 \%$, and $0.75 \%$, for the $100-, 300-$, and $1200-\mathrm{ms}$ SOAs) than for words $(M \mathrm{~s}=1.37 \%, 1.44 \%$, and $1.07 \%$, for the 100-, 300-, and 1200-ms SOAs).

\section{Typing Session}

In the typing session (see Figure 6B), the PRP effect was apparent for words and nonwords, and the effect was larger for nonwords than for words; RT2 between the 100- and 1200-ms SOAs was $286 \mathrm{~ms}$ for nonwords and $211 \mathrm{~ms}$ for words. The PRP effect also increased with string length. At the 100-ms SOA, the difference in RT2 between three- and five-letter words was $93 \mathrm{~ms}$ (46 ms/letter), and the difference between three- and five-letter nonwords was $198 \mathrm{~ms}$ (99 ms/letter). The results with words are consistent with Experiment 1.

There were a number of significant effects in RT1, IKSI, PE1, and PE2, most of which were related to differences between typing words and nonwords. RT1 was longer for nonwords ( $M=764 \mathrm{~ms})$ than for words $(M=648 \mathrm{~ms})$. The effect of string length on RT1 was also larger for nonwords than for words: on average, the 


\section{A. Lexical Decision}

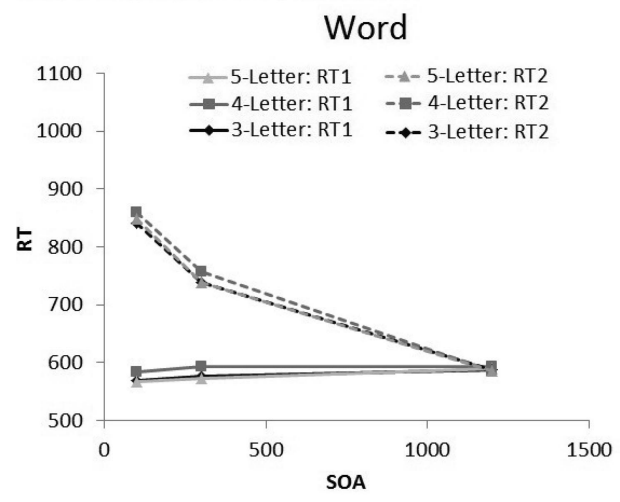

B. Typing

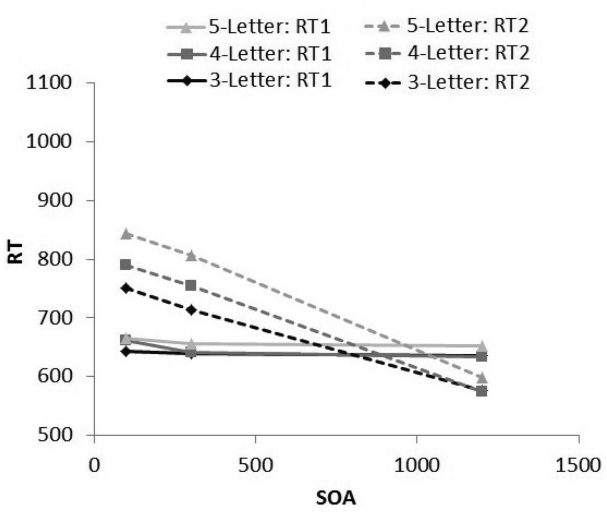

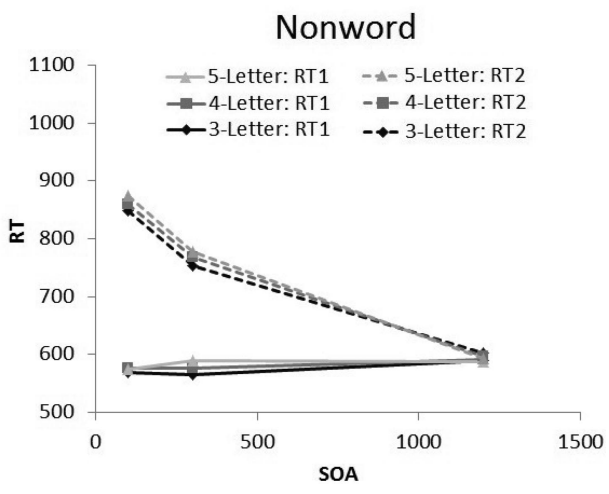

Nonword

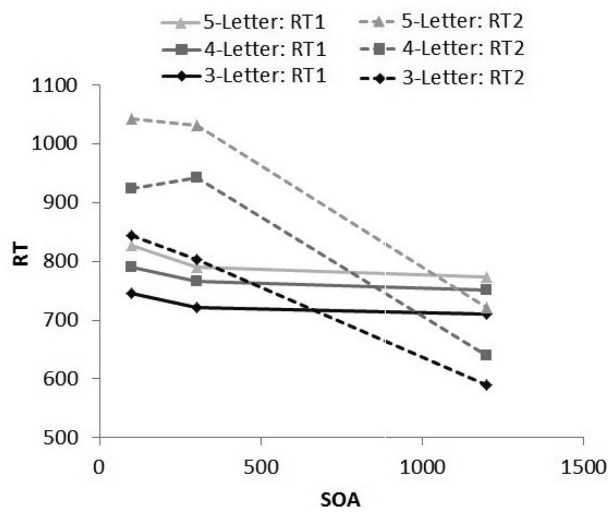

Figure 6. Response times for Task1 (RT1) and Task2 (RT2) in the Lexical Decision Session (A) and the Typing (B) Session of Experiment 2 (SOA = stimulus onset asynchrony).

increase was about $9 \mathrm{~ms} / \mathrm{letter}$ for words $(M \mathrm{~s}=640,646$, and 658 $\mathrm{ms}$, for three, four, and five letters), similar to Experiment 1. For nonwords, RT1 increased by $36 \mathrm{~ms} / \mathrm{letter}(M \mathrm{~s}=726,769$, and 797 $\mathrm{ms}$, for three-, four-, and five-letter words). In addition, RT1 was longer for shorter SOAs $(M \mathrm{~s}=722,703$, and $693 \mathrm{~ms}$, for the $100-$, 300-, and 1200-ms SOAs), indicating a task reversal effect (Miller et al., 2009): the reversal effect was larger for nonwords than for words (difference in RT1 between the 100-ms SOA and the 1200-ms SOA was 43 for nonwords and $16 \mathrm{~ms}$ for words), as the proportion of trials for which $\mathrm{R} 2$ occurred before the first keystroke was also larger for nonwords $(M=26 \%)$ than for words $(M=15 \%)$ at the 100-ms SOA (see Figure 7).

IKSI was longer for nonwords ( $M=183 \mathrm{~ms} /$ keystroke) than for words ( $M=136 \mathrm{~ms} /$ keystroke; see Table 1$)$. IKSI increased with string length for nonwords $(M \mathrm{~s}=171,183$, and $197 \mathrm{~ms}$, for three, four, and five letters; $13 \mathrm{~ms} /$ keystroke/letter) but not for words $(M \mathrm{~s}=134,134$, and $139 \mathrm{~ms} ; 2 \mathrm{~ms} /$ keystroke/letter $)$. The PE1 data indicated that typing errors occurred more frequently for nonwords than for words $(12.73 \%$ vs. $5.37 \%$, respectively; see Table 2). Typing errors also increased with string length, and the increase was larger for nonwords $(M \mathrm{~s}=8.02 \%, 10.26 \%$, and $19.90 \%$ for three, four, and five letters) than for words $(3.55 \%, 5.27 \%$, and $7.30 \%$ for three, four, and five letters). Furthermore, typing errors occurred more frequently at short SOAs than at long SOAs for nonwords $(M \mathrm{~s}=14.24 \%, 13.29 \%, 10.65 \%$, for the $100-, 300-$, and 1200-ms SOAs) but not for words $(M \mathrm{~s}=5.01 \%, 5.56 \%, 5.56 \%)$. The PE2 data indicated that errors in R2 occurred more frequently following nonwords $(M=2.21 \%)$ than following words $(M=$ $0.90 \%$; see Table 3). There was a three-way interaction among Stimulus Type, String Length, and SOA, which does not seem to reflect any systematic effect of the factors (see Table 3 ).

Overall, the outcomes of the typing session showed that the results of Experiment 1 were replicated for word trials: The PRP effect depended on the number of letters to be typed, and the increase of the effect (46 ms/letter) was smaller than IKSI (136 ms/keystroke), suggesting that typing was interruptible. The proportions of trials for which R2 occurred before the respective trials are summarized in Figure 7, which also suggest that R2 could occur anywhere between the first and the last keystrokes. The mixture proportion, estimated from Equation 3, was $35 \%$. Typing performance was generally slower for nonwords, but the string length effect ( $99 \mathrm{~ms} / \mathrm{letter})$ was smaller than IKSI (183 ms/keystroke), suggesting that typists could also interrupt nonword typing. The mixture proportion, estimated from Equation 3, was 54\%. The larger mixture proportion may reflect the larger string length effect in RT1 for nonword trials, because the string length effect in RT2 depends on the string 
Table 5

ANOVA Results of the Lexical Decision Session of Experiment 2

\begin{tabular}{|c|c|c|c|c|}
\hline Factor & $d f$ & $F$ & $M S E$ & $\eta_{p}^{2}$ \\
\hline \multicolumn{5}{|l|}{ RT1 } \\
\hline Stimulus type (ST) & 1,15 & $<1$ & 4,972 & .001 \\
\hline String length (SL) & 2,30 & 2.96 & 840 & .165 \\
\hline Stimulus onset asynchrony & 2.30 & $535^{*}$ & 1243 & 263 \\
\hline $\mathrm{ST} \times \mathrm{SL}$ & 2,30 & 2.54 & 632 & .145 \\
\hline $\mathrm{ST} \times \mathrm{SOA}$ & 2,30 & $<1$ & 1,141 & .007 \\
\hline $\mathrm{SL} \times \mathrm{SOA}$ & 4,60 & $<1$ & 807 & .029 \\
\hline $\mathrm{ST} \times \mathrm{SL} \times \mathrm{SOA}$ & 4,60 & $<1$ & 1,071 & .041 \\
\hline \multicolumn{5}{|l|}{ RT2 } \\
\hline ST & 1,15 & $6.08^{*}$ & 2,634 & .288 \\
\hline SL & 2,30 & 2.36 & 974 & .136 \\
\hline SOA & 2,30 & $61.66^{* *}$ & 27,526 & .804 \\
\hline $\mathrm{ST} \times \mathrm{SL}$ & 2,30 & 1.88 & 1,040 & .111 \\
\hline $\mathrm{ST} \times \mathrm{SOA}$ & 2,30 & 1.01 & 793 & .063 \\
\hline $\mathrm{SL} \times \mathrm{SOA}$ & 4,60 & 2.01 & 722 & .118 \\
\hline $\mathrm{ST} \times \mathrm{SL} \times \mathrm{SOA}$ & 4,60 & $<1$ & 1,273 & .044 \\
\hline \multicolumn{5}{|l|}{ PE1 } \\
\hline ST & 1,15 & 1.53 & 153 & .093 \\
\hline SL & 2,30 & $8.61^{* *}$ & 8.84 & .365 \\
\hline SOA & 2,30 & 2.06 & 16.60 & .121 \\
\hline $\mathrm{ST} \times \mathrm{SL}$ & 2,30 & 1.97 & 23.3 & .116 \\
\hline $\mathrm{ST} \times \mathrm{SOA}$ & 2,30 & $<1$ & 13.61 & .010 \\
\hline $\mathrm{SL} \times \mathrm{SOA}$ & 4,60 & 2.50 & 14.95 & .143 \\
\hline $\mathrm{ST} \times \mathrm{SL} \times \mathrm{SOA}$ & 4,60 & $<1$ & 15.95 & .042 \\
\hline \multicolumn{5}{|l|}{ PE2 } \\
\hline ST & 1,15 & 2.19 & 11.74 & .127 \\
\hline SL & 2,30 & $<1$ & 6.16 & .010 \\
\hline SOA & 2,30 & $4.95^{*}$ & 9.44 & .248 \\
\hline $\mathrm{ST} \times \mathrm{SL}$ & 2,30 & $<1$ & 3.02 & .058 \\
\hline $\mathrm{ST} \times \mathrm{SOA}$ & 2,30 & $6.14^{*}$ & 5.03 & .290 \\
\hline $\mathrm{SL} \times \mathrm{SOA}$ & 4,60 & 1.76 & 3.93 & .105 \\
\hline $\mathrm{ST} \times \mathrm{SL} \times \mathrm{SOA}$ & 4,60 & 1.20 & 4.24 & .074 \\
\hline
\end{tabular}

Note. $\quad \mathrm{RT}=$ response time; $\mathrm{IKSI}=$ interkeystroke interval; $\mathrm{PE}=$ percentage error.

${ }^{*} p<.05 .{ }^{* * *} p<.01$

length effect in RT1, but the string length effect in IKSI does not.

\section{Discussion}

The outcome of the present experiment is straightforward: There was no influence of string length on RT1 or RT2 when Task1 required lexical decision. The PRP effect was apparent, but RT2 was similar among the three string lengths at short SOAs. The results imply that word encoding, lexical access, and response selection in lexical decision do not depend on the number of letters. Hence, the results excluded the possibility that the string length effect on RT2 in the typing session were attributable to outer loop processes.

The present experiment confirmed the results of Experiment 1 when typing was Task1. There was a string length effect for RT1 and a larger string length effect for RT2. These data suggest that the influence of string length on the outer loop does not explain the string length effect on RT2, supporting the inner-loop locus. It is not clear from the present experiment whether the string-length effect on RT1 stems from the prebottleneck processes or whether there exists another bottleneck in the outer loop. Experiments 3 and 4 address this issue.

\section{Experiment 3}

Experiment 3 attempted to separate the contributions of the outer loop and inner loop by preexposing the word or nonword to be typed so that outer loop processing would be finished before typists had to begin typing. Skilled typists were presented with a word or nonword $\mathrm{S} 1$ for $1,000 \mathrm{~ms}$ but were asked to refrain typing it until a go signal appeared 2,000 ms after its onset. S2 (tone) occurred with variable SOA with respect to the onset of the go signal following S1. In Experiment 2, RT1 was less than $650 \mathrm{~ms}$ for words and less than $750 \mathrm{~ms}$ for nonwords, so 2,000 ms should be sufficient for subjects to encode $\mathrm{S} 1$ and prepare keystrokes (Balota \& Chumbley, 1985). If the string length arises from the outer loop, the effect should be eliminated entirely in RT1 and RT2. However, if the string length arises in the inner loop, the effect should still appear RT1 and RT2.

Table 6

ANOVA Results of the Typing Session of Experiment 2

\begin{tabular}{|c|c|c|c|c|}
\hline \multirow[b]{2}{*}{ Factor } & \multicolumn{4}{|c|}{ Typing } \\
\hline & $d f$ & $F$ & $M S E$ & $\eta_{p}^{2}$ \\
\hline \multicolumn{5}{|l|}{ RT1 } \\
\hline Stimulus type (ST) & 1,15 & $140.24^{* *}$ & 6,954 & .903 \\
\hline String length (SL) & 2,30 & $33.15^{* *}$ & 1,456 & .688 \\
\hline \multicolumn{5}{|l|}{ Stimulus onset asynchrony } \\
\hline (SOA) & 2,30 & $7.39^{*}$ & 2,881 & .330 \\
\hline $\mathrm{ST} \times \mathrm{SL}$ & 2,30 & $25.90^{* *}$ & 675 & .633 \\
\hline $\mathrm{ST} \times \mathrm{SOA}$ & 2,30 & $4.30^{*}$ & 1,067 & .223 \\
\hline $\mathrm{SL} \times \mathrm{SOA}$ & 4,60 & $<1$ & 669 & .038 \\
\hline $\mathrm{ST} \times \mathrm{SL} \times \mathrm{SOA}$ & 4,60 & $<1$ & 801 & .046 \\
\hline \multicolumn{5}{|l|}{ IKSI } \\
\hline ST & 1,15 & $142.68^{* *}$ & 1,147 & .905 \\
\hline SL & 2,30 & $14.09^{* *}$ & 406 & .484 \\
\hline SOA & 2,30 & $<1$ & 1,184 & .013 \\
\hline $\mathrm{ST} \times \mathrm{SL}$ & 2,30 & $20.94^{* *}$ & 130 & .583 \\
\hline $\mathrm{ST} \times \mathrm{SOA}$ & 2,30 & $<1$ & 207 & .016 \\
\hline $\mathrm{SL} \times \mathrm{SOA}$ & 4,60 & $<1$ & 168 & .037 \\
\hline $\mathrm{ST} \times \mathrm{SL} \times \mathrm{SOA}$ & 4,60 & $<1$ & 92 & .033 \\
\hline \multicolumn{5}{|l|}{ RT2 } \\
\hline ST & 1,15 & $128.12^{* *}$ & 1,693 & .895 \\
\hline SL & 2,30 & $20.94^{* * *}$ & 318 & .583 \\
\hline SOA & 2,30 & $43.99^{* * *}$ & 41,038 & .746 \\
\hline $\mathrm{ST} \times \mathrm{SL}$ & 2,30 & $26.64^{* *}$ & 3,039 & .640 \\
\hline $\mathrm{ST} \times \mathrm{SOA}$ & 2,30 & $8.30^{* *}$ & 7,799 & .356 \\
\hline $\mathrm{SL} \times \mathrm{SOA}$ & 4,60 & $7.27^{* *}$ & 2,455 & .326 \\
\hline $\mathrm{ST} \times \mathrm{SL} \times \mathrm{SOA}$ & 4,60 & $<1$ & 2,196 & .054 \\
\hline \multicolumn{5}{|l|}{ PE1 } \\
\hline ST & 1,15 & $50.16^{* *}$ & 77.61 & .770 \\
\hline SL & 2,30 & $31.37^{* *}$ & 50.5 & .676 \\
\hline SOA & 2,30 & 3.29 & 20.19 & .178 \\
\hline $\mathrm{ST} \times \mathrm{SL}$ & 2,30 & $15.47^{* * *}$ & 32.10 & .508 \\
\hline $\mathrm{ST} \times \mathrm{SOA}$ & 2,30 & $3.49^{*}$ & 30.27 & .189 \\
\hline $\mathrm{SL} \times \mathrm{SOA}$ & 4,60 & $3.13^{*}$ & 17.07 & .173 \\
\hline $\mathrm{ST} \times \mathrm{SL} \times \mathrm{SOA}$ & 4,60 & 1.90 & 25.67 & .112 \\
\hline \multicolumn{5}{|l|}{ PE2 } \\
\hline ST & 1,15 & $8.75^{* *}$ & 14.24 & .368 \\
\hline SL & 2,30 & 1.95 & 6.71 & .115 \\
\hline SOA & 2,30 & 2.88 & 6.77 & .161 \\
\hline $\mathrm{ST} \times \mathrm{SL}$ & 2,30 & 1.61 & 6.68 & .097 \\
\hline $\mathrm{ST} \times \mathrm{SOA}$ & 2,30 & 1.13 & 4.66 & .070 \\
\hline $\mathrm{SL} \times \mathrm{SOA}$ & 4,60 & 1.72 & 5.03 & .103 \\
\hline $\mathrm{ST} \times \mathrm{SL} \times \mathrm{SOA}$ & 4,60 & $3.41^{*}$ & 3.80 & .185 \\
\hline
\end{tabular}

Note. $\mathrm{RT}=$ response time; IKSI $=$ interkeystroke interval; $\mathrm{PE}=$ percentage error. ${ }^{*} p<.05 .{ }^{* * *} p<.01$. 
3-letter
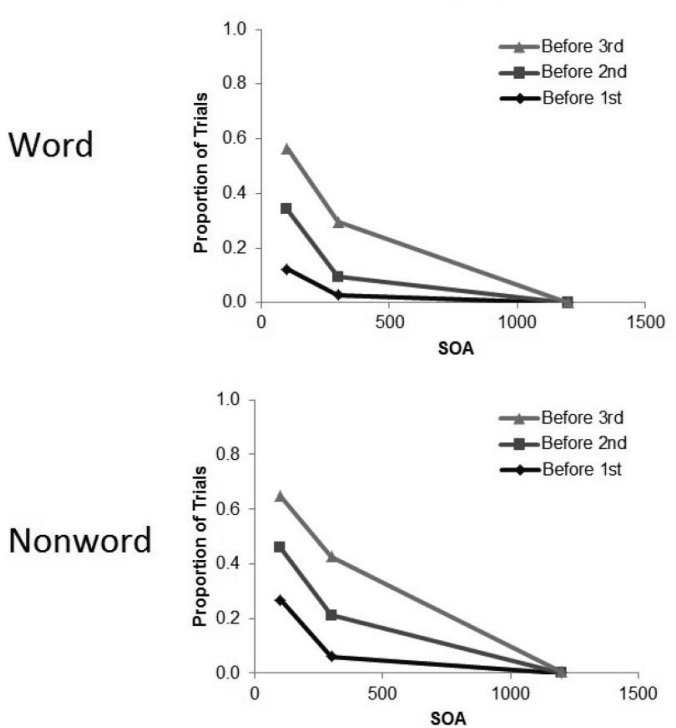

4-letter
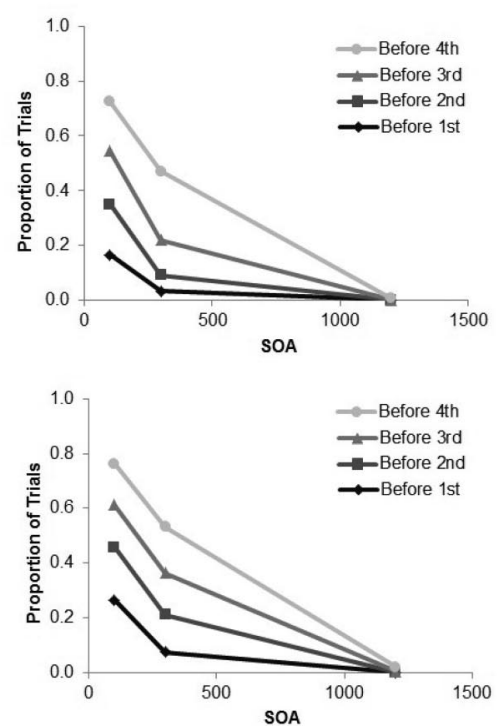

5-letter
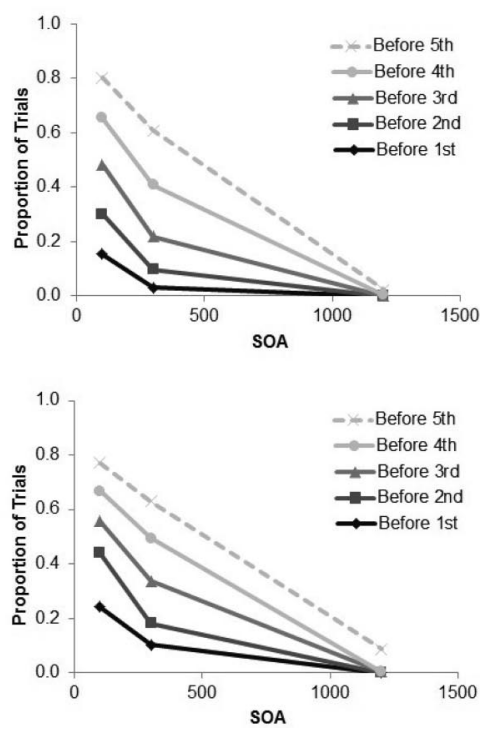

Figure 7. Proportions of trials for which R2 preceded the respective keystrokes for three-, four-, and five-letter words and nonwords in the Typing Session of Experiment 2 (SOA $=$ stimulus onset asynchrony).

Method

\section{Participants}

Sixteen touch-typists were newly recruited from the same subject pool as in the preceding experiments (13 females; mean age $=$ $18.69, S D=1.54)$. Each typist participated in a single experimental session. Mean typing speed was $82.94 \mathrm{WPM}(S D=17.25)$, and mean accuracy was $94.66 \%(S D=4.33)$. They had 5.25 months of formal training on average $(S D=1.89)$ and 11.00 years of typing experiences $(S D=1.70)$. They reported spending 2.90 hours per day for typing $(S D=1.05)$.

\section{Apparatus, Stimuli, and Procedure}

The apparatus and stimuli were identical with those of the typing session of Experiment 2. The procedure also followed the typing session of that experiment with the following changes: On each trial, a word or nonword appeared at the center of screen for $1,000 \mathrm{~ms}$, but subjects were instructed to withhold response. A red cross replaced the stimulus. Within $1,000 \mathrm{~ms}$, the red cross blinked twice (the cross was presented for $250 \mathrm{~ms}$, erased for the next 250 $\mathrm{ms}$, reappeared for $250 \mathrm{~ms}$, and then erased for $250 \mathrm{~ms}$ ). Then, the go signal "GO!" appeared at the center of screen. Subjects were instructed to start typing as soon as the go signal occurred. With a variable delay from the onset of the go signal (i.e., SOA), a highor low-pitch tone was presented through headphones. These tones were identical with those used in the preceding experiments. Vocal responses were collected in the same manner as in Experiment 2. The experimental conditions consisted of three SOAs (100, 300, and $1200 \mathrm{~ms}$ ) and three string lengths (three-, four-, and fiveletters) for word and nonword trials. The session consisted of four test blocks of 135 trials each.

\section{Results}

Because RT1 was generally much shorter in the present experiment than those in the preceding experiments, trials were excluded if RT1 was shorter than $50 \mathrm{~ms}$ or RT2 was shorter than $200 \mathrm{~ms}(<0.87 \%)$. Mean RT1 and RT2 are summarized in Figure 8, and IKSI, PE1, and PE2 are given in Tables 1, 2, and 3, respectively. The data were submitted to ANOVAs with Stimulus Type (word vs. nonword), String Length (three, four, and five letters), and SOA (100, 300, 1200 ms) as factors. The results are summarized in Table 7.

There was the PRP effect for nonwords and words, and the effect was larger for nonwords than for words (see Figure 8). The difference in RT2 between the 100- and 1200-ms SOAs was 146 ms for nonwords and $93 \mathrm{~ms}$ for words; these values are much smaller than the values obtained in Experiment 2 (286 and $211 \mathrm{~ms}$ for nonwords and words, respectively). However, the PRP effect still depended on string length: RT2 increased by $92 \mathrm{~ms} / \mathrm{letter}$ for nonwords and $35 \mathrm{~ms} / \mathrm{letter}$ for words at the 100-ms SOA; these values are remarkably similar to those obtained in Experiment 2 (99 and $46 \mathrm{~ms} /$ letter for nonwords and words, respectively).

RT1 was also shorter for words $(M=355 \mathrm{~ms})$ than for nonwords $(M=394 \mathrm{~ms}$; see Figure 8$)$. RT1 was also shorter for shorter SOAs than for longer SOAs $(M \mathrm{~s}=359,372$, and $392 \mathrm{~ms}$, for the 100-, 300-, and 1200-ms SOAs), suggesting some effect of response grouping. RT1 did not increase with string length for words $(M \mathrm{~s}=352,356$, and $355 \mathrm{~ms}$, for three, four, and five letters), but it did increase with string length for nonwords ( $M \mathrm{~s}=$ 365,387 , and $429 \mathrm{~ms}$ ). The increase for nonwords was $32 \mathrm{~ms} /$ keystroke on average, which is similar to the increase obtained in Experiment 2 (36 ms/keystroke). Therefore, the majority of the string length effect for nonwords is attributable to processes subsequent to encoding and lexical access. IKSI was also longer for nonwords $(M=142 \mathrm{~ms} /$ keystroke $)$ than for words $(M=126$ 

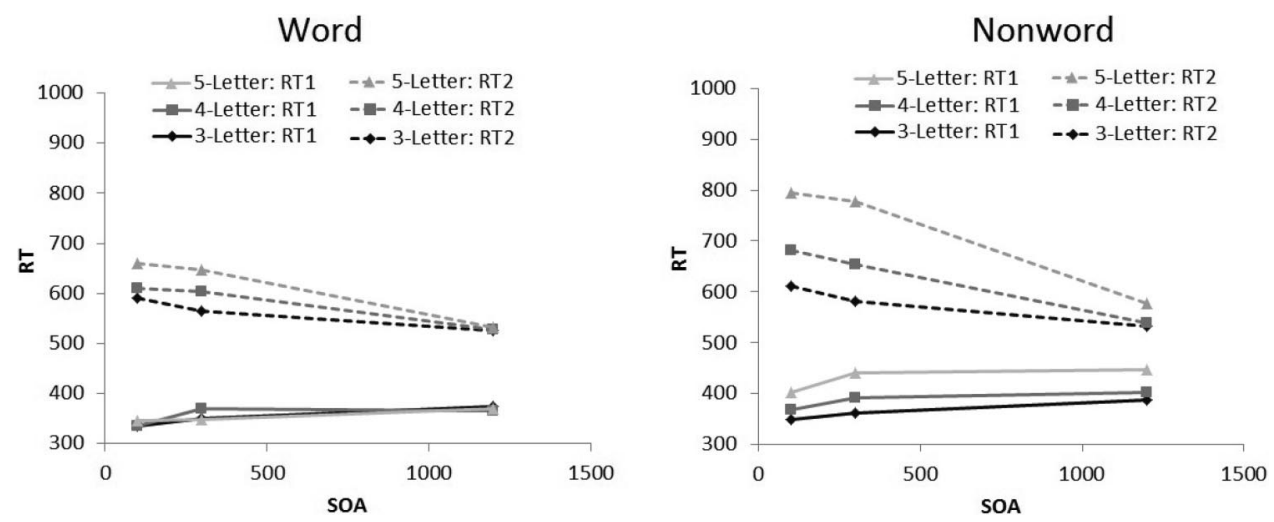

Figure 8. Response times for Typing Task1 (RT1) and Tone Task2 (RT2) in Experiment 3 (SOA = stimulus onset asynchrony).

$\mathrm{ms} /$ keystroke), but the values were smaller than in Experiment 2 (183 and $136 \mathrm{~ms} /$ keystroke for nonwords and words, respectively). IKSI increased for longer strings, but this effect was mainly attributable to nonwords ( $M \mathrm{~s}=19 \mathrm{~ms} /$ keystroke for nonwords; 7 $\mathrm{ms} /$ keystroke for words).

The mixture proportions estimated from Equation 3 were $67 \%$ and $28 \%$ for nonwords and words. Again, the larger mixture proportion for nonwords might reflect the larger string length effect in RT1 for nonwords. The proportions of trials for which R2 occurred before the respective keystrokes are summarized in Figure 9. These proportions were similar for nonword and word trials.

There were more typing errors (PE1) for longer words $(M \mathrm{~s}=$ $2.15 \%, 4.87 \%$, and $8.92 \%$, for three, four, and five letters) and for longer nonwords $(M \mathrm{~s}=2.37 \%, 5.21 \%$, and $11.00 \%$; see Table 2). PE1 tended to be larger for longer words, but the effect was marginal (the main effect of String Length had $p=.051$ ).

\section{Discussion}

The present results corroborate and extend the findings in Experiments 1 and 2 . In the present experiment, RT1 was much shorter than RT1 in the typing session of Experiment 2 (compare Figures 6 and 8), indicating that typists took advantage of the preexposure procedure to prepare themselves for typing words and nonwords. The PRP effect was reduced with the preexposure of $\mathrm{S} 1$, but the string length effect remained largely intact, increasing $36 \mathrm{~ms} / \mathrm{letter}$ for words and $93 \mathrm{~ms} / \mathrm{letter}$ for nonwords. These values were similar to those obtained in the typing session of Experiment 2 (46 and $99 \mathrm{~ms} / \mathrm{letter}$ for words and nonwords, respectively). RT1 was also reduced with preexposure, but the string length effect was eliminated only for words and not for nonwords. The string length effect for nonwords was $32 \mathrm{~ms} /$ keystroke in RT1, which is comparable with that obtained in Experiment 2 ( $36 \mathrm{~ms} / \mathrm{keystroke})$. The outcomes might mean that the current procedure excluded the contribution of the outer loop successfully for words but not for nonwords, possibly because the outer loop has to operate for each keystroke of a nonword. We discuss possible explanations and implications of these findings further in the General Discussion. Experiment 4 provides a more direct test that addresses the issue of whether the string-length effect on typing RT stems from a bottleneck process in the outer loop.

\section{Experiment 4}

In the preceding three experiments, typing was performed only as Task1. In Experiment 4, the order of the two tasks was reversed, so that the typing task was administered as Task2. Reversing task order allowed us to determine whether the string length effect in typing the first letter (RT1 in the previous experiments) constitutes a bottleneck by assessing the interaction between string length and SOA when typing was Task2.

Task1 factors can influence RT2 if they affect bottleneck or prebottleneck processes of Task1, but not if they affect postbottleneck processes. It can be determined whether the factors affect bottleneck or prebottleneck processes by reversing task order. When task order is reversed, factors that affect the prebottleneck processes should have subadditive effects with SOA on RT2 (i.e., the effects on RT2 should be smaller at shorter SOAs), but factors that affect the bottleneck process should have additive or superadditive effects with SOA (i.e., the effects should be constant across SOAs or larger at shorter SOAs). Superadditive effects may suggest resource sharing (Navon \& Miller, 2002; Pashler, 1984; Tombu \& Jolicoeur, 2003) or response grouping (Ulrich \& Miller, 2008). Hence, if string length affects prebottleneck processes, then string length should interact subadditively with SOA. If string length affects bottleneck process, string length and SOA should have additive or superadditive effects on RT2.

Subadditive effects of string length and SOA would suggest that there is only one bottleneck in typing performance, namely, the inner-loop bottleneck that selects keystrokes as typing unfolds in time. However, additive or superadditive effects would suggest there are at least two bottlenecks in typing, an outer-loop bottleneck that selects the word and an inner-loop bottleneck that selects subsequent keystrokes as typing unfolds in time.

\section{Method}

\section{Participants}

A new group of 16 touch-typists were recruited from the same subject pool (10 females; mean age $=21.69, S D=2.98)$. Mean typing speed was 75.40 WPM $(S D=18.28)$, and mean accuracy was $93.79 \%(S D=2.58)$. They had 4.25 months of formal training in typing $(S D=12.90)$ and 12.90 years of typing 
Table 7

ANOVA Results For Experiment 3

\begin{tabular}{|c|c|c|c|c|}
\hline Factor & $d f$ & $F$ & $M S E$ & $\eta_{p}^{2}$ \\
\hline \multicolumn{5}{|l|}{ RT1 } \\
\hline Stimulus type (ST) & 1,15 & $29.11^{* *}$ & 3,834 & .660 \\
\hline String length (SL) & 2,30 & $11.97^{* *}$ & 2,225 & .444 \\
\hline \multicolumn{5}{|c|}{ Stimulus onset asynchrony } \\
\hline$(\mathrm{SOA})$ & 2,30 & $22.24^{* * *}$ & 1,343 & .597 \\
\hline $\mathrm{ST} \times \mathrm{SL}$ & 2,30 & $17.55^{* *}$ & 1,392 & .539 \\
\hline $\mathrm{ST} \times \mathrm{SOA}$ & 2,30 & 1.15 & 570 & .071 \\
\hline $\mathrm{SL} \times \mathrm{SOA}$ & 4,60 & $<1$ & 1,038 & .057 \\
\hline $\mathrm{ST} \times \mathrm{SL} \times \mathrm{SOA}$ & 4,60 & 2.21 & 565 & .128 \\
\hline \multicolumn{5}{|l|}{ IKSI } \\
\hline ST & 1,15 & $64.40^{* *}$ & 289 & .811 \\
\hline SL & 2,30 & $22.33^{* *}$ & 753 & .598 \\
\hline SOA & 2,30 & 1.39 & 435 & .085 \\
\hline $\mathrm{ST} \times \mathrm{SL}$ & 2,30 & $27.30^{* * *}$ & 135 & .645 \\
\hline $\mathrm{ST} \times \mathrm{SOA}$ & 2,30 & 2.17 & 103 & .126 \\
\hline $\mathrm{SL} \times \mathrm{SOA}$ & 4,60 & $<1$ & 67 & .040 \\
\hline $\mathrm{ST} \times \mathrm{SL} \times \mathrm{SOA}$ & 4,60 & $2.61^{*}$ & 76 & .148 \\
\hline \multicolumn{5}{|l|}{ RT2 } \\
\hline ST & 1,15 & $41.81^{* *}$ & 5,162 & .736 \\
\hline SL & 2,30 & $33.32^{* *}$ & 7,013 & .690 \\
\hline SOA & 2,30 & $29.64^{* *}$ & 13,167 & .664 \\
\hline $\mathrm{ST} \times \mathrm{SL}$ & 2,30 & $17.94^{* *}$ & 2,662 & .545 \\
\hline $\mathrm{ST} \times \mathrm{SOA}$ & 2,30 & $14.22^{* *}$ & 1,372 & .487 \\
\hline $\mathrm{SL} \times \mathrm{SOA}$ & 4,60 & $17.60^{* *}$ & 1,750 & .540 \\
\hline $\mathrm{ST} \times \mathrm{SL} \times \mathrm{SOA}$ & 4,60 & $3.65^{* *}$ & 1,123 & .196 \\
\hline \multicolumn{5}{|l|}{ PE1 } \\
\hline ST & 1,15 & 4.50 & 50.14 & .231 \\
\hline SL & 2,30 & $43.35^{* *}$ & 42.86 & .743 \\
\hline SOA & 2,30 & $<1$ & 14.05 & .044 \\
\hline $\mathrm{ST} \times \mathrm{SL}$ & 2,30 & 2.07 & 27.19 & .121 \\
\hline $\mathrm{ST} \times \mathrm{SOA}$ & 2,30 & 2.39 & 21.04 & .137 \\
\hline $\mathrm{SL} \times \mathrm{SOA}$ & 4,60 & $<1$ & 16.80 & .028 \\
\hline $\mathrm{ST} \times \mathrm{SL} \times \mathrm{SOA}$ & 4,60 & $<1$ & 24.34 & .038 \\
\hline \multicolumn{5}{|l|}{ PE2 } \\
\hline ST & 1,15 & $<1$ & 3.64 & .000 \\
\hline SL & 2,30 & 2.40 & 5.61 & .138 \\
\hline SOA & 2,30 & 2.92 & 8.11 & .163 \\
\hline $\mathrm{ST} \times \mathrm{SL}$ & 2,30 & $<1$ & 10.67 & .050 \\
\hline $\mathrm{ST} \times \mathrm{SOA}$ & 2,30 & $<1$ & 4.12 & .004 \\
\hline $\mathrm{SL} \times \mathrm{SOA}$ & 4,60 & 1.05 & 7.62 & .065 \\
\hline $\mathrm{ST} \times \mathrm{SL} \times \mathrm{SOA}$ & 4,60 & $<1$ & 4.70 & .049 \\
\hline
\end{tabular}

Note. $\mathrm{RT}=$ response time; IKSI $=$ interkeystroke interval; $\mathrm{PE}=$ percentage error.

${ }^{*} p<.05 .{ }^{* * *} p<.01$.

experiences $(S D=3.00)$. They reported spending 4.94 hours per day for typing $(S D=2.01)$.

\section{Apparatus, Stimuli, and Procedure}

The apparatus and stimuli were identical with those of the typing session in Experiment 2, and the procedure also followed that session, except that Task1 and Task2 were switched. Thus, each trial started with the fixation cross, and $500 \mathrm{~ms}$ later a tone occurred, to which subjects responded by speaking into the headset microphone. At a variable SOA after the tone, a word or nonword appeared on the screen, and subjects started typing it as quickly as they could. The experimental conditions consisted of three SOAs $(100,300$, and $1200 \mathrm{~ms}$ ) and three string lengths (three, four, and five letter) for word and nonword trials. The session was separated into six test blocks of 90 trials each and lasted for less than an hour.

\section{Results}

Trials for which RT1 or RT2 were less than 200 ms were excluded from the analysis $(<0.93 \%)$. Mean RT1, RT2, IKSI, PE1, and PE2 were submitted to separate ANOVAs as a function of Stimulus Type (word vs. nonword), String Length (three, four, and five letters), and SOA $(100,300,1200 \mathrm{~ms})$; the results are summarized in Table 8. RT1 and RT2 are plotted in Figure 10, and IKSI, PE1, and PE2 are summarized in Tables 1, 2, and 3, respectively. R2 occurred before R1 less than $1 \%$ of trials at the shortest SOA, so we do not discuss the proportion of task-reversal in the present experiment.

The PRP effect was obtained for both words and nonwords (see Figure 10). The effect was larger for nonwords than for words (the differences in RT2 between the 100- and 1200-ms were 89 and $65 \mathrm{~ms}$ for nonwords and words, respectively). There was also an effect of string length on RT2 $(M \mathrm{~s}=15$ and $11 \mathrm{~ms} / \mathrm{letter}$ for nonwords and words). Importantly, the string-length effect was larger at short SOAs (Ms $=19,15$, and $4 \mathrm{~ms} / \mathrm{letter}$ for the 100-, 300-, and 1200-ms SOAs). This superadditive interaction implies that the process that produces the string-length effect in typing RT constitutes a bottleneck. In general the PRP effect appears to be smaller than those obtained when typing was performed as Task1. This outcome may occur because the prebottleneck processes for typing are much longer than the prebottleneck processes for tone discrimination, so there is relatively short queuing time for typing when it is performed as Task2.

RT2 was longer for nonwords $(M=767 \mathrm{~ms})$ than for words $(M=663 \mathrm{~ms})$, and the difference between words and nonwords was larger at shorter SOAs than for longer SOAs $(M \mathrm{~s}=113,109$, and 89 ms, for the 100-, 300-, and 1200-ms SOAs; see Figure 10). Thus, stimulus type also interacted with SOA superadditively, implying that the process that produces the stimulus type effect also constitutes a bottleneck in typing.

RT1 was longer for shorter SOAs $(M \mathrm{~s}=563,539$, and $536 \mathrm{~ms}$, for the 100-, 300-, and 900-ms SOAs; see Figure 10), which may reflect capacity sharing between two tasks (Navon \& Miller, 2002; Tombu \& Jolicœur, 2003) or response grouping (Ulrich \& Miller, 2008) that occurred at the 100-ms SOA. The outcome may also reflect task reversal, but this explanation is less likely to hold in the present experiment because, as noted earlier, R2 occurred before $\mathrm{R} 1$ less than $1 \%$ of trials at the shortest SOA. There was a tendency for RT1 to increase with string length for words ( $M=8 \mathrm{~ms} / \mathrm{letter})$ but not for nonwords ( $M=-3 \mathrm{~ms} / \mathrm{letter})$.

IKSI was longer for nonwords than for words, and it increased with string length for nonwords ( $M=15 \mathrm{~ms} /$ keystroke/letter) but not for words ( $M=2 \mathrm{~ms} /$ keystroke/letter; see Table 1). IKSI was longer for shorter SOAs than for longer SOAs $(M \mathrm{~s}=152,151$, and $144 \mathrm{~ms} /$ keystroke from the 100-, 300-, and 1200-ms SOAs).

There were no effects in PE1. Typing error rates (PE2) were higher for nonwords $(M=9.64 \%)$ than for words $(M=4.91 \%)$, for longer strings $(M \mathrm{~s}=5.81 \%, 6.55 \%$, and $9.46 \%$, for three, four, and five letters), and shorter SOAs (Ms $=7.69 \%, 8.00 \%$, and $6.13 \%$, for the 100-, 300-, and 1200-ms SOAs; see Table 3).

\section{Discussion}

The present experiment examined whether the effect of string length on typing RT stemmed from prebottleneck or bottleneck processes by looking at how the effect interacted with SOA. The results were clear: string length interacted with SOA superadditively. The superadditivity may be attributable to response grouping (Ulrich \& 

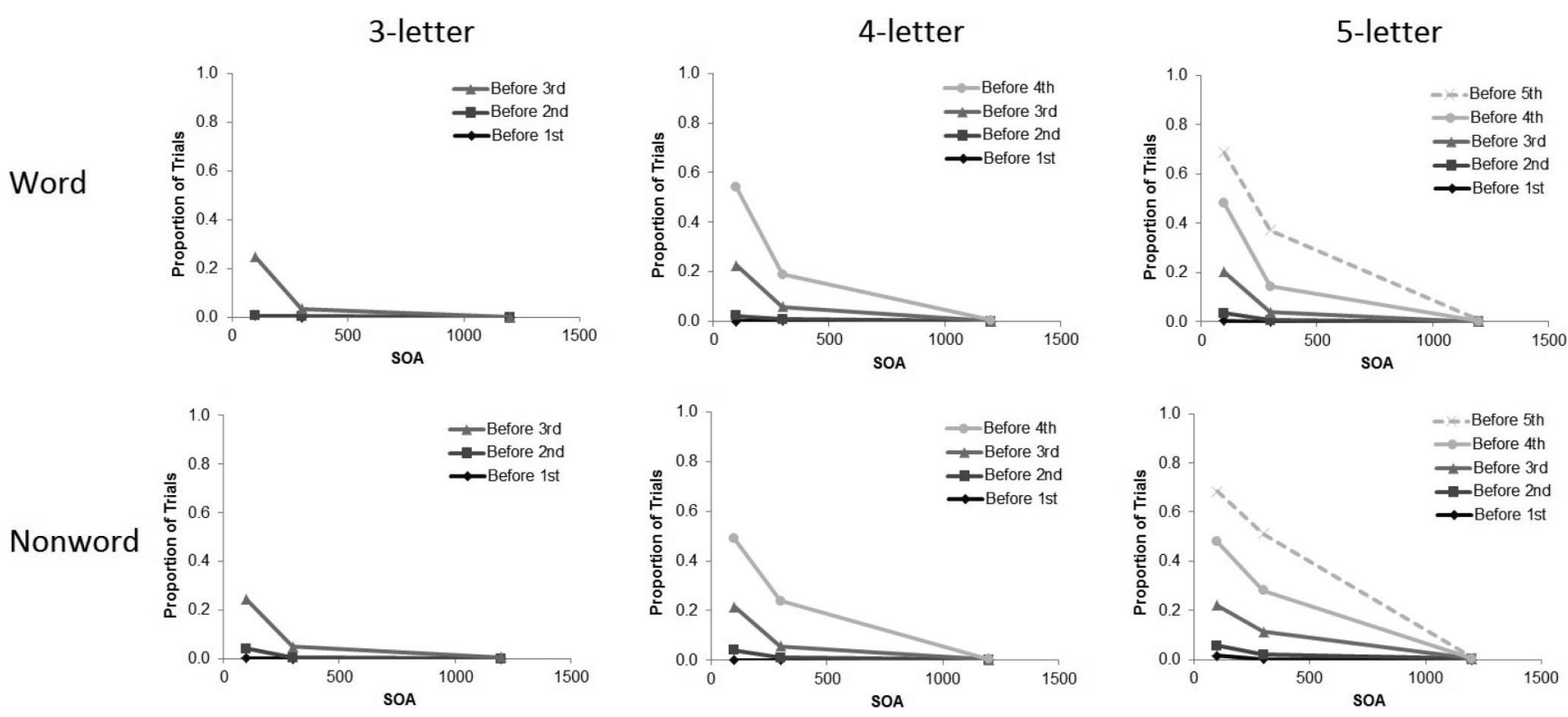

Figure 9. Proportions of trials for which R2 preceded the respective keystrokes for three-, four-, and five-letter words and nonwords in Experiment 3 (SOA = stimulus onset asynchrony).

Miller, 2008) or to resource sharing between typing and vocal response required for tone discrimination (Navon \& Miller, 2002; Pashler, 1984; Tombu \& Jolicoer, 2003) because two tasks share the verbal component of working memory (e.g., Hayes \& Chenoweth, 2006). In either case, the results imply that the effect stems from a bottleneck process in typing. In the preceding three experiments where typing was performed as Task1, we observed that the string length affected both RT1 and RT2, and the effect was stronger in RT2 than in RT1, suggesting two different loci for string-length effects. We suggested that one locus is in the inner loop, and the results of Experiments 2 and 3 supported our conjecture. In the present experiment, we found that the string-length effect in typing RT also constitutes a bottleneck. Consequently, there exist bottlenecks in both inner and outer loop processing in typing.

\section{General Discussion}

The present study investigated whether response selection in skilled typewriting selects words or letters. We used the PRP paradigm to answer this question, manipulating word length when typing was Task1. If response selection selects words, RT2 should not depend on word length because only one response is selected on each trial. If response selection selects letters (or keystrokes), RT2 should increase with word length because one response would be selected for each letter. Experiments 1-3 found that RT2 increased with word length at short SOAs. Therefore, response selection in skilled typewriting selects letters.

Theories of skilled typewriting suggest that typing performance involves hierarchically organized control processes (Logan \& Crump, 2011; Rumelhart \& Norman, 1982; Sternberg et al., 1990). The higher level outer loop controls the word-level processing, which encodes a word and passes it to the lower level inner loop, which implements individual keystrokes. In this theoretical framework, the conclusion that response selection selects letters means that a bottleneck exists in the inner loop.
String length also affected the latency of the first keystroke (RT1). However, the effect of word length on RT1 was smaller than the effect on RT2, indicating that the effect on RT1 cannot fully account for the effect on RT2. Hierarchical theories of typing suggest that typing RT involves the latency of the outer loop and the latency of the inner loop, whereas IKSI involves only the latency of the inner loop (Logan \& Crump, 2011). There was no effect of string length on IKSI when typing words, implying that the string-length effect on RT1 reflects outer loop processing.

In Experiment 4, we reversed the order of tasks, so that typing was performed as Task2, and we found that string length interacted with SOA superadditively, implying that the string length effect on typing RT reflects bottleneck processing in the outer loop (Navon \& Miller, 2002; Pashler, 1984; Tombu \& Jolicoeur, 2003). Therefore, the results of the four experiments indicate that there are bottlenecks in the outer loop and in the inner loop.

\section{Interruption in Skilled Typing}

We compared three formal models of the inner-loop locus of the PRP effect in the present study. The first model assumed that typing is uninterruptible, so that all keystrokes need to be selected before the bottleneck is allocated to Task2. This model predicted that RT2 at short SOAs would increase as much as IKSI per additional letter in a word (Equation 1). The second model assumed that typing can be interrupted at the completion of the bottleneck process for a keystroke, and the model predicted an increase of RT2 at short SOAs that was smaller than IKSI (Equation 2). The third model assumed that typing is a mixture of uninterruptible and interruptible cases (Equation 3). Throughout Experiments 1-3, the data were consistent with the interruptible model and the mixture model.

The increases in RT2 at the 100-ms SOA were about $20-30 \%$ of IKSI for words and 50-60\% for nonwords. The larger ratios for nonwords may imply that typing nonwords are more difficult to 
Table 8

ANOVA Results for Experiment 4

\begin{tabular}{|c|c|c|c|c|}
\hline Factor & $d f$ & $F$ & $M S E$ & $\eta_{p}^{2}$ \\
\hline \multicolumn{5}{|l|}{ RT1 } \\
\hline Stimulus type (ST) & 1,15 & $<1$ & 535 & .055 \\
\hline String length (SL) & 2,30 & 1.26 & 421 & .078 \\
\hline Stimulus onset asynchrony (SOA) & 2,30 & $8.31^{* * *}$ & 2,604 & .357 \\
\hline $\mathrm{ST} \times \mathrm{SL}$ & 2,30 & $3.83^{*}$ & 977 & .203 \\
\hline $\mathrm{ST} \times \mathrm{SOA}$ & 2,30 & $<1$ & 695 & .069 \\
\hline $\mathrm{SL} \times \mathrm{SOA}$ & 4,60 & 1.11 & 717 & .069 \\
\hline $\mathrm{ST} \times \mathrm{SL} \times \mathrm{SOA}$ & 4,60 & 1.53 & 520 & .092 \\
\hline \multicolumn{5}{|l|}{ RT2 } \\
\hline ST & 1,15 & $105.03^{* * *}$ & 7,391 & .875 \\
\hline SL & 2,30 & $7.64^{* *}$ & 2,249 & .337 \\
\hline SOA & 2,30 & $32.49^{* *}$ & 4,361 & .684 \\
\hline $\mathrm{ST} \times \mathrm{SL}$ & 2,30 & $<1$ & 1,734 & .061 \\
\hline $\mathrm{ST} \times \mathrm{SOA}$ & 2,30 & $5.14^{*}$ & 765 & .255 \\
\hline $\mathrm{SL} \times \mathrm{SOA}$ & 4,60 & $3.73^{* *}$ & 597 & .199 \\
\hline $\mathrm{ST} \times \mathrm{SL} \times \mathrm{SOA}$ & 4,60 & 1.13 & 582 & .070 \\
\hline \multicolumn{5}{|l|}{ IKSI } \\
\hline ST & 1,15 & $76.70^{* *}$ & 1,755 & .836 \\
\hline SL & 2,30 & $43.55^{* *}$ & 149 & .744 \\
\hline SOA & 2,30 & $8.83^{* *}$ & 200 & .371 \\
\hline $\mathrm{ST} \times \mathrm{SL}$ & 2,30 & $33.28^{* * *}$ & 120 & .689 \\
\hline $\mathrm{ST} \times \mathrm{SOA}$ & 2,30 & 2.91 & 121 & .162 \\
\hline $\mathrm{SL} \times \mathrm{SOA}$ & 4,60 & $<1$ & 94 & .044 \\
\hline $\mathrm{ST} \times \mathrm{SL} \times \mathrm{SOA}$ & 4,60 & 2.29 & 67 & .132 \\
\hline \multicolumn{5}{|l|}{ PE1 } \\
\hline ST & 1,15 & 3.66 & 5.27 & .196 \\
\hline SL & 2,30 & $<1$ & 5.27 & .059 \\
\hline SOA & 2,30 & 2.20 & 5.33 & .128 \\
\hline $\mathrm{ST} \times \mathrm{SL}$ & 2,30 & $<1$ & 3.07 & .042 \\
\hline $\mathrm{ST} \times \mathrm{SOA}$ & 2,30 & 2.82 & 1.30 & .158 \\
\hline $\mathrm{SL} \times \mathrm{SOA}$ & 4,60 & $<1$ & 2.34 & .045 \\
\hline $\mathrm{ST} \times \mathrm{SL} \times \mathrm{SOA}$ & 4,60 & 1.73 & 2.93 & .103 \\
\hline \multicolumn{5}{|l|}{ PE2 } \\
\hline ST & 1,15 & $39.85^{* *}$ & 40.46 & .727 \\
\hline SL & 2,30 & $18.41^{* *}$ & 19.36 & .551 \\
\hline SOA & 2,30 & $4.85^{*}$ & 19.97 & .244 \\
\hline $\mathrm{ST} \times \mathrm{SL}$ & 2,30 & $<1$ & 23.86 & .003 \\
\hline $\mathrm{ST} \times \mathrm{SOA}$ & 2,30 & $<1$ & 16.56 & .002 \\
\hline $\mathrm{SL} \times \mathrm{SOA}$ & 4,60 & 1.98 & 30.26 & .117 \\
\hline $\mathrm{ST} \times \mathrm{SL} \times \mathrm{SOA}$ & 4,60 & $<1$ & 27.43 & .035 \\
\hline
\end{tabular}

Note. $\mathrm{RT}=$ response time; IKSI $=$ interkeystroke interval; $\mathrm{PE}=$ percentage error.

${ }^{*} p<.05{ }^{* *} p<.01$.

interrupt, or they may reflect the large word length effect on RT1 in these trials, which increased the word length effect on RT2 but not IKSI. In nonword trials of Experiment 2, there were word length effects of $36 \mathrm{~ms} / \mathrm{letter}$ in RT1 and $99 \mathrm{~ms} / \mathrm{letter}$ in RT2 at the 100-ms SOA. After subtracting the effect in RT1 from the effect in RT2, we obtained the "corrected" mixture proportion of $34 \%$. In nonword trials of Experiment 3, there were word length effects of $32 \mathrm{~ms} / \mathrm{letter}$ in RT1 and $92 \mathrm{~ms} / \mathrm{letter}$ in RT2. The corrected mixture proportion was about $42 \%$. Thus, the mixture proportions for nonwords still appear larger than those for words, implying that interruption is less likely for nonwords than for words. Possibly, interruption is less likely for nonwords because typing nonwords requires more effort (to maintain and rehearse letters to be typed in short-term memory or to monitor keystrokes being executed) than typing words, so typists are more likely to postpone Task2. Further investigations are needed to address this issue.

Notwithstanding, the present study supports the conclusion that skilled typing is rather easily interrupted, which corroborates the finding that skilled typing can be interrupted whenever necessary (Logan, 1982) and extend it to conditions in which typing has to be resumed after the interruption. When the typing task was performed as Task1, IKSI was somewhere between 130 and $140 \mathrm{~ms}$. These values are no larger than IKSI obtained in previous studies in which typing is performed without concurrent tasks (e.g., see Crump \& Logan, 2010a; Logan \& Crump, 2009; Yamaguchi, Crump, \& Logan, in press) or in Experiment 4 of the present study in which typing was performed as Task2 (IKSI was about 120-130 $\mathrm{ms})$. The outcomes suggest that even if typing is interrupted, resumption can occur smoothly.

\section{What Does the Inner-Loop Bottleneck Do?}

In the PRP literature, response selection is often identified with the process of translating stimulus to response (e.g., McCann \& Johnston, 1992; Pashler, 1984; Welford, 1952). This would imply that response selection in the inner loop translates letters into keystrokes. However, there are reasons to doubt that this is the case. For instance, Crump and Logan (2010a) found that pressing a key on the keyboard was faster when the corresponding letter appeared in a word that was presented visually or acoustically before the imperative stimulus occurred. This priming effect did not occur when the letter appeared in a string of random letters. When it occurred in a word, the position of the letter in the word did not affect the magnitude of the priming effect (except the first position). These results suggest that all letters in a word are translated into keystrokes before the first keystroke is executed. Also, Logan (2003) found that when a to-be-typed word is presented on the left or right of the screen, typing RT was shorter when there were more keystrokes in a word that were executed by the hand (left or right hand) compatible with the word location in the screen. This Simon-type effect implies that all letters are translated into keystrokes before the first key is pressed. Electrophysiological evidence also supports parallel activation of keystrokes, which shows a larger cortical activation in a hemisphere at the first keystroke when there are more letters that are executed by the hand on the side contralateral to that hemisphere (i.e., the hand controlled by that hemisphere; Logan et al., 2011).

In Rumelhart and Norman's (1982) model of skilled typewriting, keystroke selection occurs continuously and in parallel for all letters in a word. In the model, each keystroke is represented by a keystroke schema, and all relevant schemata get activated when a word is encoded. Activation of the schema is translated into the actual movement of the corresponding finger toward the key, and when the finger reaches the key location, it only needs to move downward and depress the key. The bottleneck may start with the onset of the finger movement and end at the point in which the finger reaches the key location. During this period, the model assumes that all subsequent keystroke schemata receive inhibition from the first keystroke schema. It might be this inhibitory process that also suppresses R2 until the current keystroke schema completes (i.e., until the finger is positioned above the key).

Previous studies used a high-speed video camera to observe finger movements during typing (Flanders \& Soeching, 1992; McLeod \& Hume, 1994) and found that the finger movements for successive keystrokes can overlap temporally, demonstrating parallel execution of the keystrokes. However, McLeod and Hume also found that the finger usually starts moving within $\pm 40 \mathrm{~ms}$ of 

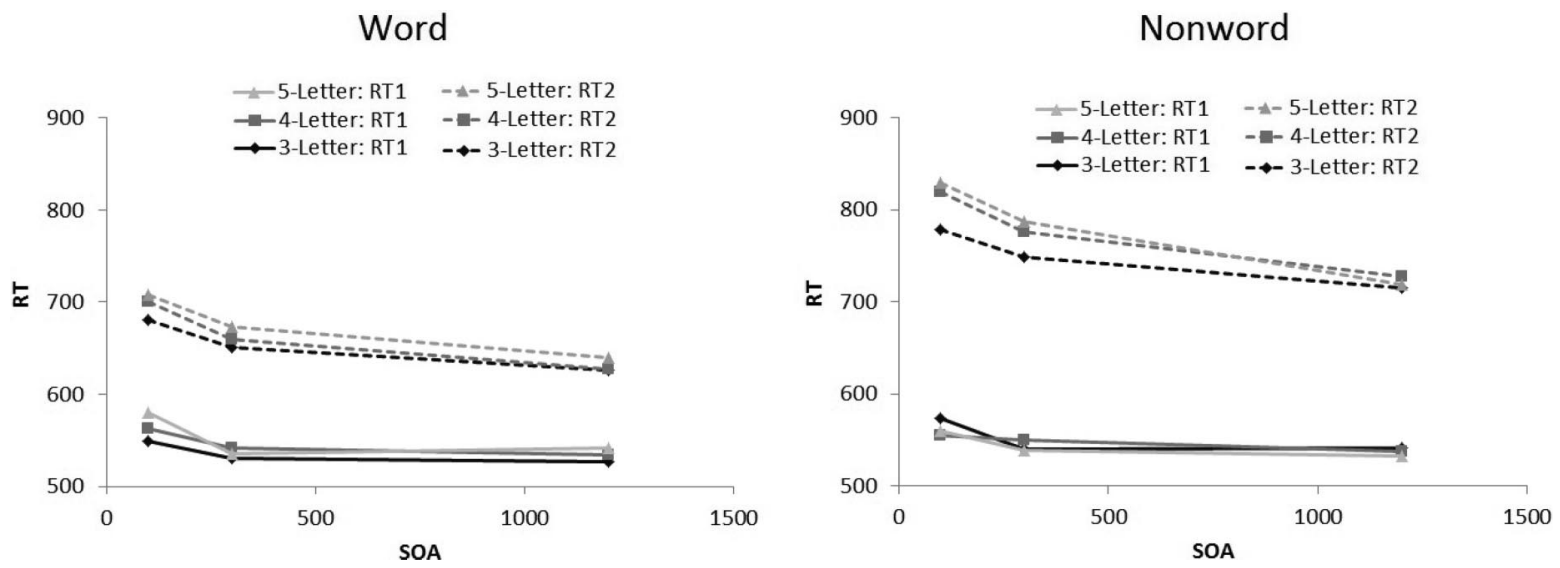

Figure 10. Response times for Tone Task1 (RT1) and Typing Task2 (RT2) in Experiment 4 (SOA = stimulus onset asynchrony).

the striking of a preceding keystroke, which suggested to them that the launch time of a keystroke is not distributed randomly but closely tied to the execution of the preceding keystroke. They proposed that a bottleneck exists in typing to control execution of successive keystrokes.

Several researchers have suggested that a bottleneck exists in response initiation or execution. For instance, Logan and Burkell (1986) found that the PRP effect occurred only when subjects made R1 but not when they inhibited R1. De Jong (1993) found a smaller PRP effect when R1 was not required than when R1 was required. He also found that the manipulation of response selection complexity (whether Task2 required choice reaction or simple reaction) interacted with SOA subadditively, implying that there is a bottleneck that follows the response-selection process. Pashler and Christian (1994) found that when Task1 required moving a finger toward a visual stimulus, RT2 was correlated not only with RT1 (initiation of the aimed movement) but also with the movement time of R1, which appeared consistent with the response-execution bottleneck. Ferreira and Pashler (2002) suggested that RT2 also depended on a very late processing component of speech production that Task1 required. More recently, Ulrich et al. (2006) used R1 that required a guided ballistic movement of an arm (moving a lever along a track) and found that the PRP effect depended on the duration of the arm movement (also see Bratzke et al., 2008). These findings suggest the existence of a bottleneck that is closely related to response execution. Our finding of the inner-loop locus of the PRP effect is consistent with these previous findings.

Another possible function of the inner-loop bottleneck is monitoring of response feedback (Welford, 1968). Jentzsch, Leuthold, and Ulrich (2007) proposed that the propagation of an execution-related latency of Task1 to RT2 could be attributable to response monitoring that consumes central resources (but see Bratzke, Rolke, \& Ulrich, 2009). The two-loop theory of skilled typewriting (Logan \& Crump, 2011) proposes that the outer loop and the inner loop monitor distinct sources of feedback (see Figure 1); the outer loop monitors typed texts on the screen whereas the inner loop monitors kinesthetic or haptic feedback on the fingers (also see, e.g., Barrett \& Krueger, 1994; Crump \& Logan, 2010b; Logan \& Crump, 2010). This monitoring process might constitute a bottleneck in the inner loop. This possibility requires further investigation.

\section{What Does the Outer-Loop Bottleneck Do?}

The bottleneck in the outer loop may involve retrieving a lexical item that corresponds to the word, but it is not clear why the process depends on the number of letters in a string to be typed. In Experiment 2, we showed that the PRP effect was not dependent on the length of words or nonwords when Task1 was lexical decision. Also, RT1 in the lexical-decision task was not affected by that factor. In the motor control literature, it has widely been reported that more complex action sequences take longer to initiate. The phenomenon is attributed to preparation of the entire movement sequence in advance to its initiation (e.g., Henry \& Rogers, 1960; Sternberg, Knoll, Monsell, \& Wright, 1988; Sternberg, Monsell, Knoll, \& Wright, 1978). Our finding that typing RT increases with string length may be another example of this phenomenon.

Sternberg et al. (1978) proposed two stages of sequence preparation. In the first stage (program construction), a motor command is prepared by retrieving and linking a set of subprograms in a motor buffer. Construction may take longer for longer sequences because more subprograms have to be loaded into the buffer. In the second stage (subprogram retrieval), a subprogram is retrieved from the buffer to execute the first action in the sequence. Retrieval may take longer for longer sequences because there are more subprograms to maintain in the buffer, so memory scanning takes longer the longer the sequence (Sternberg, 1969a). If only the first stage is assumed, the length of action sequence would affect the latency of the first action but not the latencies of the subsequent actions. If only the second stage is assumed, the length of action sequence would affect the latencies of all actions uniformly. If both stages are assumed, the length of action sequence affects the latencies of all actions, with the first action being prolonged most because it is subject to the influences of both stages.

In the present study, the effect of string length on RT was generally smaller for words (about $10 \mathrm{~ms} / \mathrm{letter}$ ) than for nonwords (35-45 ms/letter). Loading a familiar sequence into the buffer may take less time than loading an unfamiliar sequence. Moreover, string length affected typing RT but not IKSI for words, but it affected both RT and IKSI for nonwords. Thus, with words, the string-length effect on typing RT may depend on the program 
construction; with nonwords, the string-length effect on typing RT may depend on program construction and subprogram retrieval.

With words, all keystrokes in the motor buffer may be chunked into a single entity and submitted to the inner loop at once, so no memory scanning is needed. With nonwords, individual keystrokes may be stored as separate entities and submitted one at a time, so memory scanning is needed and scanning time increases with the number of letters in the nonword. This also explains why IKSI for nonwords is longer than IKSI for words: with nonwords, IKSI includes retrieval time for execution of each keystroke, whereas with words IKSI does not include retrieval time because retrieval is not required. These conjectures are speculative, but a sequence preparation process is a good candidate for the outer-loop bottleneck.

\section{Concluding Remarks}

Typewriting has become an important survival skill in the modern society. The majority of subjects who participated in the present study reported that they spend as many as four hours in typing every day. Thus, typing occupies one quarter of their waking hours (also see Logan \& Crump, 2011). This remarkable fact suggests that typewriting is one of the most highly trained skills that can be studied with a common subject population used in psychological research.

Training makes skills automatic (e.g., Logan, 1988; Schneider \& Shiffrin, 1977), and evidence suggests that skilled typists are not consciously aware of how their fingers implement individual keystrokes (Liu, Crump, \& Logan, 2010; Logan \& Crump, 2009; Tapp \& Logan, 2011) as if the inner loop operates automatically. Many researchers claim that automatic processes bypass processing bottlenecks (e.g., Greenwald \& Shulman, 1973; McLeod \& Posner, 1984; Maquestiaux et al., 2008; Pashler, 1984; Shaffer, 1975b), so we should expect no PRP effect in skilled typing performance. Thus, it is counterintuitive to observe that bottlenecks still exist in typing performance, especially in the processes at the keystroke level.

Hierarchical control is believed to underlie many complex tasks, including playing a musical instrument (Lashley, 1951), operating a vehicle (Fitts \& Posner, 1967), or even making a cup of coffee (Cooper \& Shallice, 2000). Many of these tasks are performed on a daily basis, so people have attained high levels of proficiency in the tasks. The continuous nature of these tasks makes it difficult to determine whether they involve bottleneck processes by applying the PRP logic, but studies suggest that these tasks do produce dual-task interference (e.g., Strayer \& Johnston, 2011). Hence, we expect that the results of the present study would generalize to other hierarchical skills.

Finally, the present finding that skilled typing can be interrupted in any pair of successive keystrokes has a theoretically important implication. Through training, skilled performers gain such things as speed and accuracy, and in exchange, may lose such things as generality and flexibility of action, as automaticity of skills would imply (e.g., Anderson, 1983; Fitts \& Posner, 1967; Schneider \& Shiffrin, 1977). Do skilled performers lose control on their actions through training as well? The present study suggests they do not (see also Pashler, 1998). The finding that typing can be interrupted between any pair of successive keystrokes suggests that skilled performance as highly trained as typewriting is also subject to deliberate control (also see Logan, 1982; Yamaguchi, Crump, \& Logan, in press). Because skilled performance is so fast, it may be difficult to intervene. However, the present study suggests that training does not reduce the ability to control actions, but instead, it changes form of control that can be exercised; for example, from algorithm-based computation to instance retrieval (Logan, 1988), or executing individual component processes separately to executing a chunk of processes in parallel (Rosenbloom \& Newell, 1987). Therefore, highly trained automatic skills are not the opposite of deliberately controlled performances.

\section{References}

Anderson, J. R. (1982). Acquisition of cognitive skill. Psychological Review, 89, 369-406. doi:10.1037/0033-295X.89.4.369

Balota, D. A., \& Chumbley, J. (1985). The locus of word-frequency effects in the pronunciation task: Lexical access and/or production? Journal of Memory and Language, 24, 89-106. doi:10.1016/0749596X(85)90017-8

Barrett, J., \& Krueger, H. (1994). Performance effects of reduced proprioceptive feedback on touch typists and causal users in a typing task. Behavior \& Information Technology, 13, 373-381. doi:10.1080/ 01449299408914618

Bratzke, D., Rolke, B., \& Ulrich, R. (2009). The source of executionrelated dual-task interference: Motor bottleneck or response monitoring? Journal of Experimental Psychology: Human Perception and Performance, 35, 1413-1426. doi:10.1037/a0015874

Bratzke, D., Ulrich, R., Rolke, B., Schröter, H., Jentzsch, I., \& Leuthold, H. (2008). Motor limitation in dual-task processing with different effectors. Quarterly Journal of Experimental Psychology, 61, 1385-1399. doi: 10.1080/17470210701536856

Broadbent, D. E. (1982). Task combination and the selective intake of information. Acta Psychologica, 50, 253-290. doi:10.1016/00016918(82)90043-9

Byrne, M. D., \& Anderson, J. R. (2001). Serial modules in parallel: The psychological refractory period and perfect time-sharing. Psychological Review, 108, 847-869. doi:10.1037/0033-295X.108.4.847

Cooper, R. P., \& Shallice, T. (2000). Contention scheduling and the control of routine activities. Cognitive Neuropsychology, 17, 297-338. doi: $10.1080 / 026432900380427$

Crump, M. J. C., \& Logan, G. D. (2010a). Hierarchical control and skilled typing: Evidence for word-level control over the execution of individual keystrokes. Journal of Experimental Psychology: Learning, Memory, and Cognition, 36, 1369-1380. doi:10.1037/a0020696

Crump, M. J. C., \& Logan, G. D. (2010b). Warning: This keyboard will deconstruct-The role of the keyboard in skilled typewriting. Psychonomic Bulletin \& Review, 17, 394-399. doi:10.3758/PBR.17.3.394

De Jong, R. (1993). Multiple bottlenecks in overlapping task performance. Journal of Experimental Psychology: Human Perception and Performance, 19, 965-980. doi:10.1037/0096-1523.19.5.965

Donders, F. C. (1969). On the speed of mental processes. Acta Psychologica, 30, 412-431 (original work published in 1968). doi:10.1016/ 0001-6918(69)90065-1

Eriksen, B. A., \& Eriksen, C. W. (1974). Effects of noise letters upon the identification of a target letter in a nonsearch task. Perception \& Psychophysics, 16, 143-149. doi:10.3758/BF03203267

Fendrick, P. (1937). Hierarchical skills in typewriting. Journal of Educational Psychology, 28, 609-620. doi:10.1037/h0054049

Ferreira, V. S., \& Pashler, H. (2002). Central bottleneck influences on the processing stages of word production. Journal of Experimental Psychology: Learning, Memory, and Cognition, 28, 1187-1199. doi:10.1037/ 0278-7393.28.6.1187

Fitts, P. M., \& Posner, M. I. (1967). Human performance. Belmont, CA: Brooks/Cole.

Flanders, M., \& Soechting, J. F. (1992). Kinematics of typing: Parallel control of the two hands. Journal of Neurophysiology, 67, 1264-1274. 
Greenwald, A. G., \& Shulman, H. G. (1973). On doing two things at once: II. Elimination of the psychological refractory period effect. Journal of Experimental Psychology, 101, 70-76. doi:10.1037/h0035451

Hayes, J. R., \& Chenoweth, N. A. (2006). Is working memory involved in the transcribing and editing of texts? Written Communication, 23, 135149. doi: $10.1177 / 0741088306286283$

Henry, F. M., \& Rogers, E. E. (1960). Increased response latency fro complicated movements and a "memory drum" theory of neuromotor reaction. Research Quarterly of the American Association for Health, Physical Education, \& Recreation, 31, 448-458.

Hershman, R. L., \& Hillix, W. A. (1965). Data processing in typing: Typing rate as a function of kind of material and amount of exposed. Human Factors, 7, 483-492.

Hommel, B. (1998). Automatic stimulus-response translation in dual-task performance. Journal of Experimental Psychology: Human Perception and Performance, 24, 1368-1384. doi:10.1037/0096-1523.24.5.1368

Jentzsch, 1., Leuthold, H., \& Ulrich, R. (2007). Decomposing sources of response slowing in the PRP paradigm: The residual PRP effect. Journal of Experimental Psychology: Human Perception and Performance, 33, $610-626$

Lashley, K. S. (1951). The problem of serial order in behavior. In L. A. Jeffress (Ed.), Cerebral mechanisms in behavior (pp. 112-146). New York: Wiley

Liu, X., Cump, M. J. C., \& Logan, G. D. (2010). Do you know where your fingers have been? Explicit knowledge of the spatial layout of the keyboard in skilled typists. Memory \& Cognition, 38, 474-484. doi: 10.3758/MC.38.4.474

Logan, G. D. (1982). On the ability to inhibit complex actions: A stopsignal study of typewriting. Journal of Experimental Psychology: Human Perception and Performance, 8, 778-792. doi:10.1037/0096-1523 .8.6.778

Logan, G. D. (1988). Toward an instance theory of automatization. Psychological Review, 95, 492-527. doi:10.1037/0033-295X.95.4.492

Logan, G. D. (2003). Simon-type effects: Chronometric evidence for keypress schemata in typewriting. Journal of Experimental Psychology: Human Perception and Performance, 29, 741-757. doi:10.1037/00961523.29.4.741

Logan, G. D., \& Burkell, J. (1986). Dependence and independence in responding to double stimulation: A comparison of stop, change, and dual-task paradigms. Journal of Experimental Psychology: Human Perception and Performance, 12, 549-563. doi:10.1037/0096-1523.12.4 .549

Logan, G. D., \& Crump, M. J. C. (2009). The left hand doesn't know what the right hand is doing. Psychological Science, 20, 1296-1300. doi: 10.1111/j.1467-9280.2009.02442.x

Logan, G. D., \& Crump, M. J. C. (2010). Cognitive illusions of authorship reveal hierarchical error detection in skilled typists. Science, 330, 683686. doi:10.1126/science.1190483

Logan, G. D., \& Crump, M. J. C. (2011). Hierarchical control of cognitive processes: The case for skilled typewriting. In B. Ross (Ed.), The psychology of learning and motivation (Vol. 54, pp. 1-27). Burlington, MA: Academic Press.

Logan, G. D., \& Gordon, R. D. (2001). Executive control of visual attention in dual-task situations. Psychological Review, 108, 393-434. doi:10.1037/0033-295X.108.2.393

Logan, G. D., Miller, A. E., \& Strayer, D. L. (2011). Electrophysiological evidence for parallel response selection in skilled typists. Psychological Science, 22, 54-56. doi:10.1177/0956797610390382

Logan, G. D., \& Zbrodoff, N. J. (1998). Stroop-type interference: Congruity effects in color naming with typewritten responses. Journal of Experimental Psychology: Human Perception and Performance, 24, $978-$ 992. doi:10.1037/0096-1523.24.3.978

Maquestiaux, F., Laguë-Beauvais, M., Ruthruff, E., \& Bherer, L. (2008). Bypassing the central bottleneck after single-task practice in the psy- chological refractory period paradigm: Evidence for task automatization and greedy resource recruitment. Memory \& Cognition, 36, 1262-1282. doi:10.3758/MC.36.7.1262

McCann, R. S., \& Johnston, J. C. (1992). Locus of the single-channel bottleneck in dual-task interference. Journal of Experimental Psychology: Human Perception and Performance, 18, 471-484. doi:10.1037/ 0096-1523.18.2.471

McLeod, P., \& Hume, M. (1994). Overlapping mental operations in serial performance with preview: Typing. A reply to Pashler. Quarterly Journal of Experimental Psychology, 47A, 193-199.

McLeod, P., \& Posner, M. I. (1984). Privileged loops from percept to act. In H. Bourma and D. G. Bouwhuis (Eds.), Attention and performance X (pp. 55-66). Hillsdale, NJ: Lawrence Erlbaum.

Meyer, D. E., \& Kieras, D. E. (1997). A computational theory of executive cognitive processes and multiple-task performance: Part 2. Accounts of psychological refractory-period phenomena. Psychological Review, 104, 749-791. doi:10.1037/0033-295X.104.4.749

Miller, G. A., Galanter, E., \& Pribram, K. H. (1960). Plans and the structure of behavior. New York: Adams-Bannister-Cox.

Miller, J., Ulrich, R., \& Rolke, B. (2009). On the optimality of serial and parallel processing in the psychological refractory period paradigm: Effects of the distribution of stimulus onset asynchronies. Cognitive Psychology, 58, 273-310. doi:10.1016/j.cogpsych.2006.08.003

Navon, D., \& Miller, J. (2002). Queuing or sharing? A critical evaluation of the single-bottleneck notion. Cognitive Psychology, 44, 193-251. doi:10.1006/cogp.2001.0767

Pashler, H. (1984). Processing stages in overlapping tasks: Evidence for a central bottleneck. Journal of Experimental Psychology: Human Perception and Performance, 10, 358-377. doi:10.1037/0096-1523.10.3.358

Pashler, H. (1994a). Dual-task interference in simple tasks: Data and theory. Psychological Bulletin, 116, 220-244. doi:10.1037/0033-2909 116.2.220

Pashler, H. (1994b). Overlapping mental operations in serial performance with preview. Quarterly Journal of Experimental Psychology, 47, 161-191.

Pashler, H. (1994c). Comment on McLeod and Hume, overlapping mental operations in serial performance with preview: Typing. Quarterly Journal of Experimental Psychology, 47A, 201-205.

Pashler, H. (1998). The psychology of attention. Cambridge, MA: MIT Press.

Pashler, H., \& Christian, C. L. (1994). Bottlenecks in planning and producing vocal, manual and foot responses. Tech Report, Center for Human Information Processing.

Pashler, H., \& Johnston, J. C. (1989). Chronometric evidence for central postponement in temporally overlapping tasks. Quarterly Journal of Experimental Psychology, 41A, 19-45.

Posner, M. I., \& Snyder, C. R. R. (1975). Attention and cognitive control. In R. L. Solso (Ed.), Information processing and cognition: The Loyola Symposium (pp. 55-85). Hillsdale, NJ: Lawrence Erlbaum.

Rosenbloom, P. S., \& Newell, A. (1987). Learning by chunking: A production-system model of practice. In D. Klahr, P. Langley, \& R. Neches (Eds.), Production system models of learning and development (pp. 221-286). Cambridge, MA: MIT Press.

Rumelhart, D. E., \& Norman, D. A. (1982). Simulating a skilled typist: A study of skilled cognitive-motor performance. Cognitive Science, 6 , 1-36. doi:10.1207/s15516709 $\operatorname{cog} 0601 \_1$

Ruthruff, E., Johnston, J. C., Van Selst, M., Whitsell, S., \& Remington, R. (2003). Vanishing dual-task interference after practice: Has the bottleneck been eliminated or is it merely latent? Journal of Experimental Psychology: Human Perception and Performance, 29, 280-289. doi: 10.1037/0096-1523.29.2.280

Schneider, W., \& Shiffrin, R. M. (1977). Controlled and automatic human information processing: I. Detection, search, and attention. Psychological Review, 84, 1-66. doi:10.1037/0033-295X.84.1.1 
Shaffer, L. H. (1973). Latency mechanisms in transcription. In S. Kornblum (Ed.), Attention and performance IV (pp. 435-448). New York, NY: Academic Press

Shaffer, L. H. (1975a). Control processes in typing. Quarterly Journal of Experimental Psychology, 27, 419-432. doi:10.1080/ 14640747508400502

Shaffer, L. H. (1975b). Multiple attention in continuous verbal tasks. In. P. M. A. Rabbitt, \& S. Dornic (Eds.), Attention and performance $V$ (pp. 157-167). New York, NY: Academic Press.

Shaffer, L. H., \& Hardwick, J. (1969). Reading and typing. Quarterly Journal of Experimental Psychology, 21, 381-383. doi:10.1080/ 14640746908400233

Sternberg, S. (1969a). Memory-scanning: Mental processes revealed by reaction-time experiments. American Scientist, 57, 421-457.

Sternberg, S. (1969b). The discovery of processing stages: Extensions of Donders' method. Acta Psychologica, 30, 276-315. doi:10.1016/00016918(69)90055-9

Sternberg, S., Knoll, R. L., Monsell, S., \& Wright, C. E. (1988). Motor programs and hierarchical organization in the control of rapid speech. Phonetica, 45, 175-197. doi:10.1159/000261825

Sternberg, S., Knoll, R. L., \& Turock, D. L. (1990). Hierarchical control in the execution of action sequences: Tests of two invariance properties. In M. Jeannerod (Ed.), Attention and performance XIII (pp. 3-55). Hillsdale, NJ: Lawrence Erlbaum.

Sternberg, S., Monsell, S., Knoll, R. L., \& Wright, C. E. (1978). The latency and duration of speech and typewriting. In G. E. Stelmach (Ed.), Information processing in motor control and learning (pp. 117-152). New York, NY: Academic Press.

Strayer, D. L., \& Johnston, W. A. (2001). Driven to distraction: Dual-task studies of simulated driving and conversing on a cellular telephone. Psychological Science, 12, 462-466. doi:10.1111/1467-9280.00386

Tapp, K. M., \& Logan, G. D. (2011). Attention to the hands disrupts skilled typewriting: The role of vision in producing the disruption. Attention,
Perception, \& Psychophysics, 73, 2379-2383. doi:10.3758/s13414-0110208-5

Tombu, M., \& Jolicœur, P. (2003). A central capacity sharing model of dual-task performance. Journal of Experimental Psychology: Human Perception and Performance, 29, 3-18. doi:10.1037/0096-1523.29 .1 .3

Ulrich, R., Fernández, S. R., Jentzsch, I., Rolke, B., Schröter, H., \& Leuthold, H. (2006). Motor limitation in dual-task processing under ballistic movement conditions. Psychological Science, 17, 788-793. doi:10.1111/j.1467-9280.2006.01783.x

Ulrich, R., \& Miller, J. (2008). Response grouping in the psychological refractory period (PRP) paradigm: Models and contamination effects. Cognitive Psychology, 57, 75-121. doi:10.1016/j.cogpsych.2007.06.004

Vallacher, R. R., \& Wegner, D. M. (1987). What do people think they're doing? Action Identification and Human Behavior. Psychological Review, 94, 3-15. doi:10.1037/0033-295X.94.1.3

Van Selst, M., Ruthruff, E., \& Johnston, J. C. (1999). Can practice eliminate the psychological refractory period effect? Journal of Experimental Psychology: Human Perception \& Performance, 25, 1268 1283. doi:10.1037/0096-1523.25.5.1268

Welford, A. T. (1952). The "psychological refractory period" and the timing of high speed performance-A review and a theory. British Journal of Psychology, 43, 2-19.

Welford, A. T. (1968). Fundamentals of skill. London, UK: Methuen.

Yamaguchi, M., Crump, M. J. C., \& Logan, G. D. (in press). Speedaccuracy tradeoff in skilled typewriting: Decomposing the contributions of hierarchical control loops. Journal of Experimental Psychology: Human Perception and Performance.

Yap, M. J., Balota, D. A., Cortese, M. J., \& Watson, J. M. (2006). Singleversus dual-process models of lexical decision performance: Insights from response time distributional analysis. Journal of Experimental Psychology: Human Perception and Performance, 32, 1324-1344. doi: 10.1037/0096-1523.32.6.1324

\section{Appendix}

\section{Formal Analysis of the PRP Procedure With Typing as Task1}

If selection of each keystroke constitutes a bottleneck in typing, there are two possible cases for which Task 2 could use the bottleneck when typing is performed as Task1 of the psychological refractory period (PRP) procedure. In the first case, typing is an uninterruptible process, so that typists have to process all letters before processing Task 2 at the bottleneck. In the second case, typing is an interruptible process, such that typists can stop typing in the middle of a word, insert R2, and resume typing the remaining letters. Predictions of the bottleneck model in these two cases are considered separately below. The main goal in this section is to derive the expected effect of the number of letters in a word (string length) on the queuing time of
Task2 bottleneck processing (i.e., the PRP effect). To set up the discussions of these predictions, we start with the definitions of general constructs assumed in the bottleneck model.

\section{Assumptions and Definitions}

Typing performance can be measured in terms of reaction time (RT; the interval between word onset and the first keystroke) and interkeystroke interval (IKSI; the interval between successive keystrokes). According to the two-loop theory of skilled typewriting (Logan \& Crump, 2011), RT reflects both the outer- and inner- 


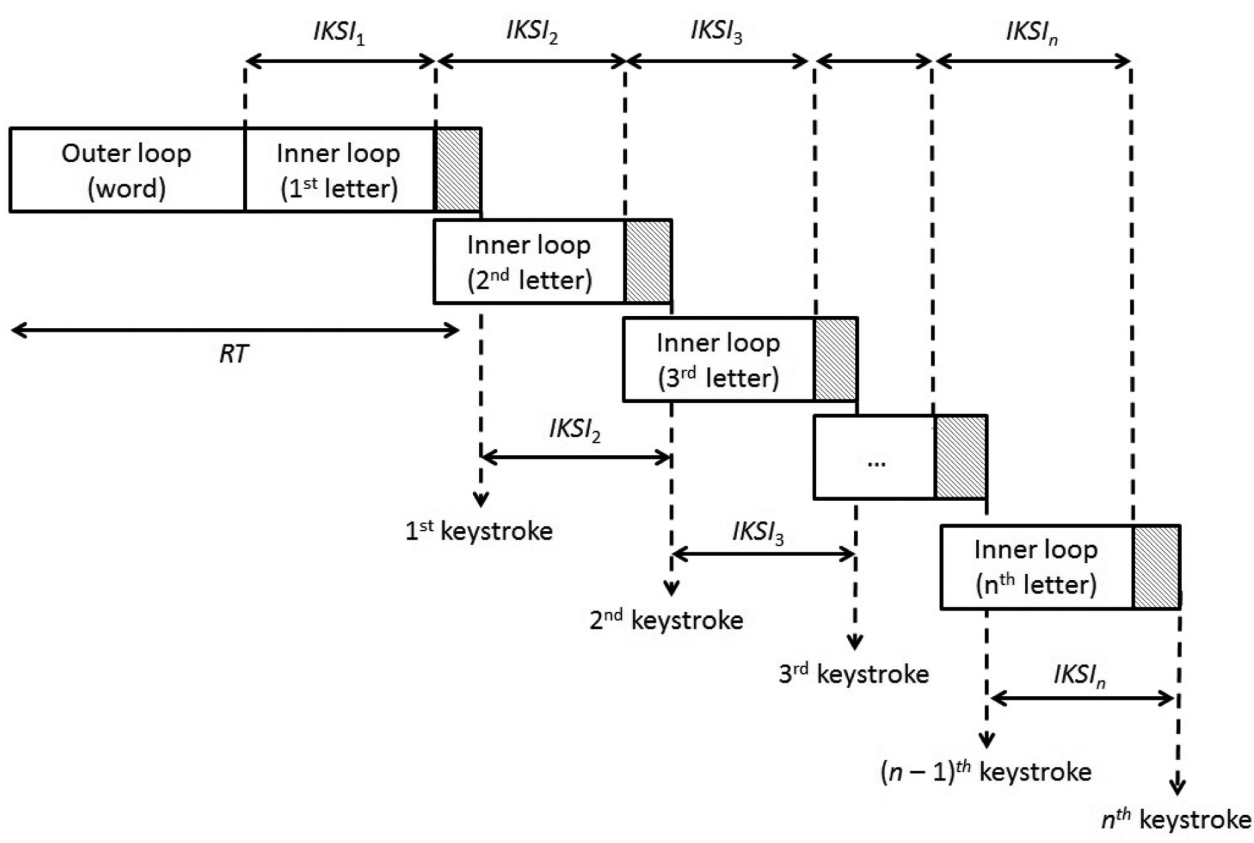

Figure A1. Illustration of the two-loop theory of skilled typewriting.

loop processing, whereas IKSI reflects the inner-loop processing. We assume that all keystrokes have been activated simultaneously upon completion of the outer-loop processing, and IKSI corresponds to the duration of the bottleneck process that is required to complete a single keystroke. As depicted in Figure A1, each inner-loop process consists of bottleneck and postbottleneck components, and if the latency of postbottleneck component is distributed identically across all keystrokes, the observed IKSI corresponds to the latency of the bottleneck component; that is,

$$
\mathrm{E}\left[I K S I_{i}\right]=E\left[B_{i}-C_{i-1}\right]+E\left[C_{i}\right]=E\left[B_{i}\right],
$$

where $B_{i}$ and $C_{i}$ are latencies of the bottleneck and postbottleneck components of the $i$ th keystroke. Thus, we disregard the postbottleneck process of each keystroke in the following discussions. The current model assumes that the bottleneck process of each keystroke starts immediately as the preceding keystroke completes the process when typing is performed alone, so the bottleneck is occupied seamlessly until all keystrokes complete it once the first keystroke starts the process.

Throughout the following discussions, we assume the central bottleneck model of the PRP task (Pashler, 1994a; Welford, 1952), which states that only a single task can be processed at the bottleneck at any given time and all other tasks have to be queued until the current process completes. In addition, we also assume that the bottleneck process runs to completion once it has started (i.e., it does not terminate in the middle of choosing a single response), and any task can start the bottleneck as soon as it completes the prebottleneck processes if no task is using it at its arrival (i.e., the first-come, first-served principle).

For Task1, let $T_{1}$ denote a random variable that represents the duration of the outer-loop operation. Also, we assume that the duration of the inner-loop operation does not depend on the position of the keystroke in a word, so the interkeystroke interval of the $i$ th keystroke, $I K S I_{i}$, is a random variable that is independently and identically distributed for all $i=1,2, \ldots, n$ (for n-letter words).

For Task2, let $T_{2}$ be a random variable that represents the duration of the prebottleneck processes. We assume that Task2 prebottleneck processing is independent of the number of letters required to type a word. Let $p_{i}^{(n)}$ designate the probability that, for $n$-letter words, Task 2 prebottleneck processes complete when the $i$ th keystroke occupies the bottleneck. Then, we write:

$$
p_{i}^{(n)}=\operatorname{Pr}\left[T_{1}+\sum_{k=1}^{i-1} I K S I_{k}<T_{2}+S O A \leq T_{1}+\sum_{k=1}^{i} I K S I_{k}\right] .
$$




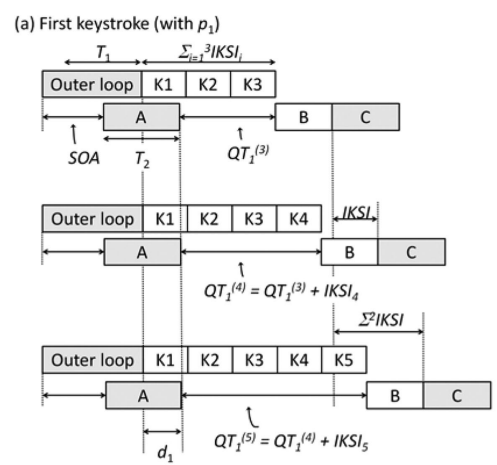

(b) Second keystroke (with $p_{2}$ )

(c) Third keystroke (with $p_{3}$ )
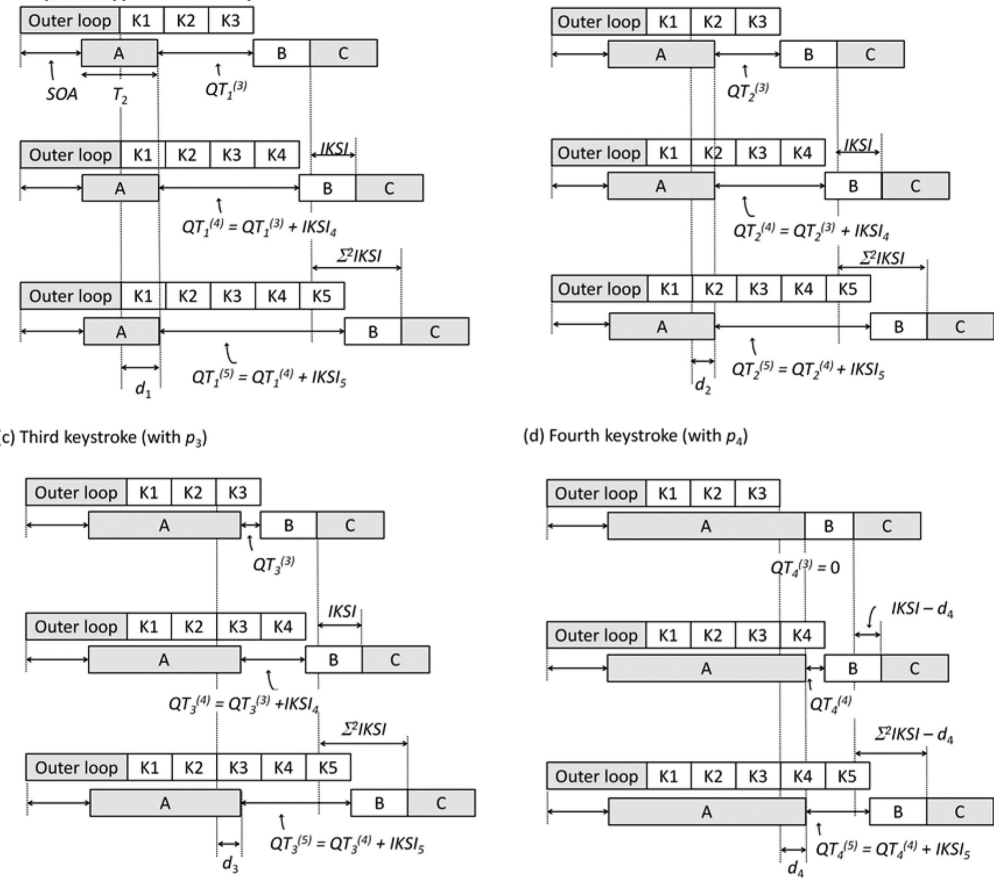

(d) Fourth keystroke (with $p_{4}$ )

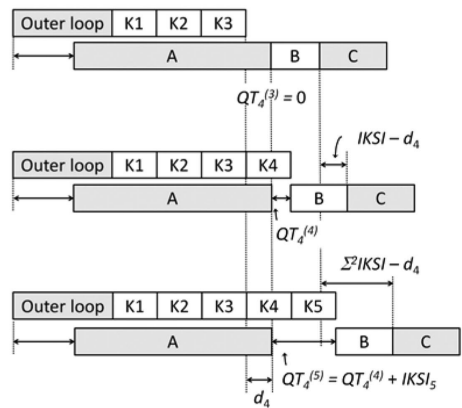

(e) Fifth keystroke (with $p_{5}$ )

(f) After all keystrokes (with $p_{6}$ )
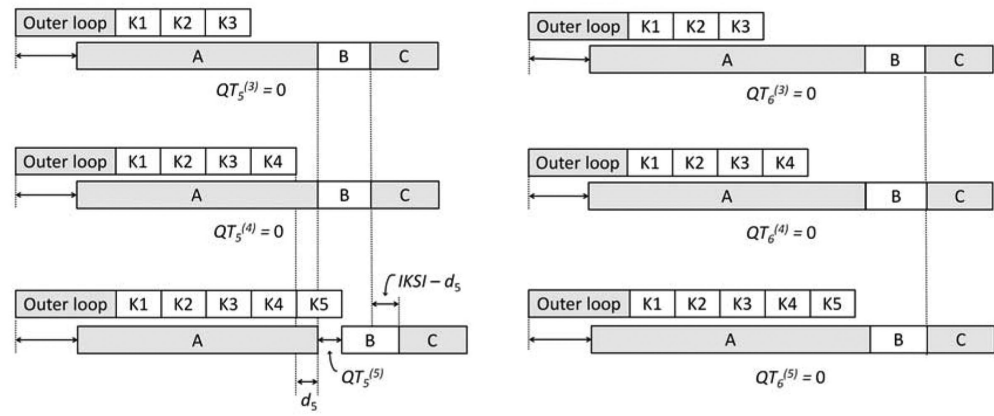

(g) Before the first keystroke (with $p_{0}$ )

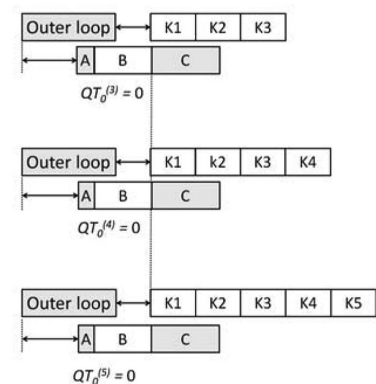

$$
\begin{aligned}
& E\left[Q T^{(3)}\right]=\sum_{i=1}^{3} p_{i} \cdot E\left[Q T_{i}^{(3)}\right] \\
& E\left[Q T^{(4)}\right]=E\left[Q T^{(3)}\right]+\sum_{i=1}^{4} p_{i} \cdot E\left[I K S I_{4}\right]-p_{4} \cdot E\left[d_{4}\right] \\
& E\left[Q T^{(5)}\right]=E\left[Q T^{(4)}\right]+\sum_{i=1}^{5} p_{i} \cdot E\left[I K S I_{5}\right]-p_{5} \cdot E\left[d_{5}\right]
\end{aligned}
$$

Figure A2. Predictions of the uninterruptible typing model in which Task2 arrives at the bottleneck while (a) the first, (b) second, (c) third, (d) fourth, or (e) fifth keystroke occupies the bottleneck, after (f) all keystrokes have completed the bottleneck process, or $(\mathrm{g})$ before the first keystroke starts the bottleneck process $(\mathrm{A}=$ Task2 pre-bottleneck; $\mathrm{B}=$ Task2 bottleneck; $\mathrm{C}=$ Task2 post-bottleneck; $\mathrm{K} 1-5=$ the first to fifth keystrokes). 

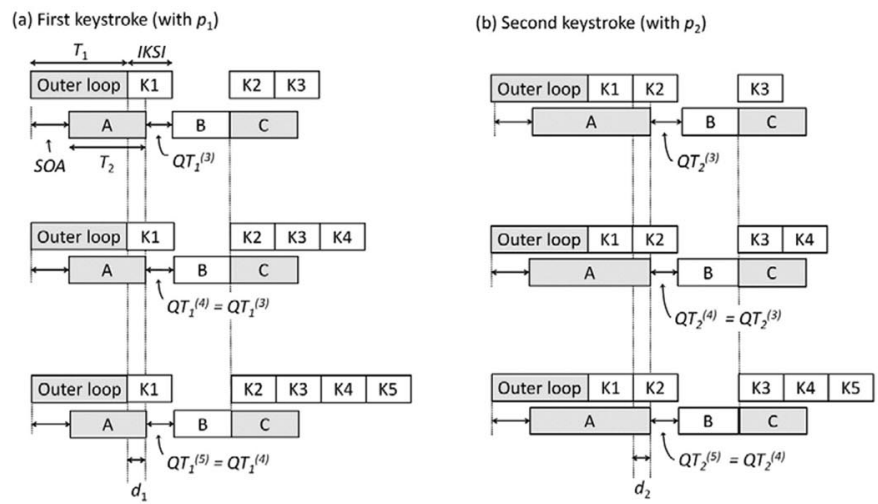

(c) Third keystroke (with $p_{3}$ )

(d) Fourth keystroke (with $p_{4}$ )
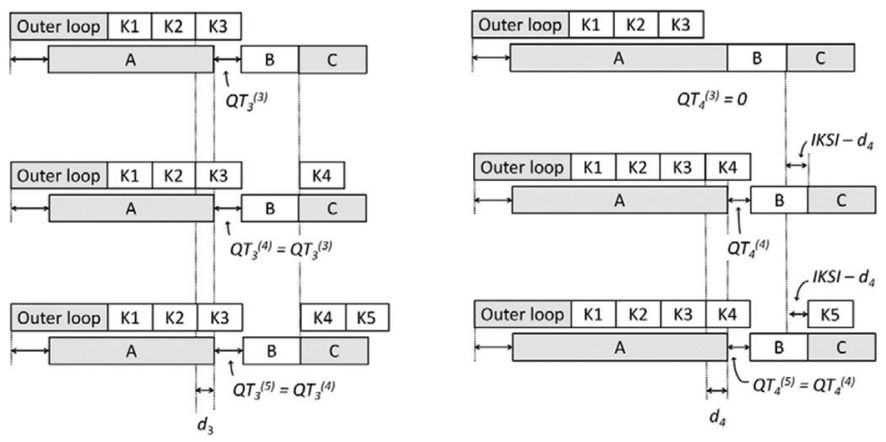

(e) Fifth keystroke (with $p_{s}$ )

(f) After all keystrokes (with $p_{6}$ )

\begin{tabular}{|l|l|l|l|}
\hline Outer loop & K1 & K2 & K3 \\
\hline
\end{tabular}

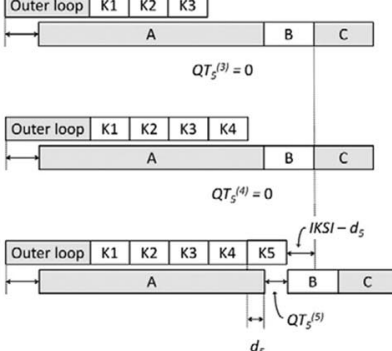

\begin{tabular}{|l|l|l|l|}
\hline Outer loop & K1 & K2 & K3 \\
\hline
\end{tabular}

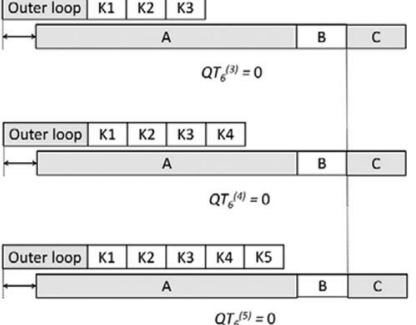

(B) Before the first keystroke (with $p_{0}$ )
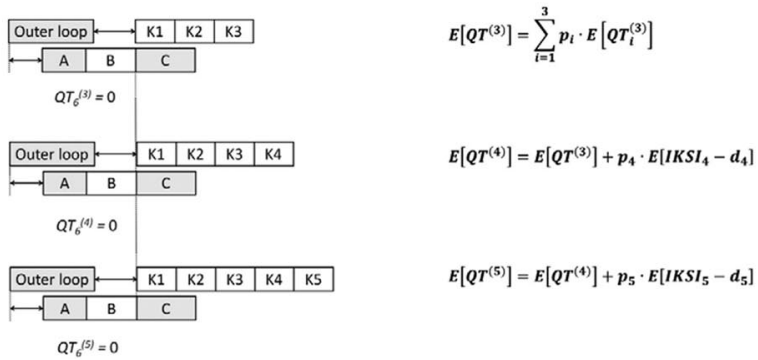

Figure A3. Predictions of the interruptible typing model in which Task2 arrives at the bottleneck while (a) the first, (b) second, (c) third, (d) fourth, or (e) fifth keystroke occupies the bottleneck, after (f) all keystrokes have completed the bottleneck process, or $(\mathrm{g})$ before the first keystroke starts the bottleneck process $(\mathrm{A}=$ Task2 pre-bottleneck; $\mathrm{B}=$ Task2 bottleneck; $\mathrm{C}=$ Task2 post-bottleneck; $\mathrm{K} 1-5=$ the first to fifth keystrokes). 
The independence of Task 2 prebottleneck from the word length manipulation implies $p_{i}^{(\mathrm{m})}=p_{i}{ }^{(n)}=p_{i}$ for any $m$ and $n$, provided that $T_{I}$ and $I K S I_{i}$ are also independent of word length. To simplify the notation, we use $p_{i}$ for all word lengths. Also, if $n$ is the longest word length used in the experiment (i.e., $N=5$ in the present study), $p_{N+1}$ is used to denote the probability that the Task2 prebottleneck completes after the $n$th keystroke has completed the process;

$$
p_{N+1}=\operatorname{Pr}\left[T_{1}+\sum_{k=1}^{N} I K S I_{k} \leq T_{2}+S O A\right] .
$$

Similarly, $p_{0}$ is used to denote the probability that the Task2 prebottleneck completes before the first keystroke has started the process;

$$
p_{0}=\operatorname{Pr}\left[T_{2}+S O A \leq T_{1}\right] .
$$

Then, we have $\left(p_{0}+p_{1}+\ldots+p_{n}+p_{n+1}\right)=1$.

\section{Uninterruptible Typing Case}

Predictions for the uninterruptible case are illustrated in Figure A2. As shown in the figure, Task 2 can start the bottleneck processing only after all keystrokes have gone through the bottleneck. Let $Q T_{i}^{(n)}$ represents the queuing time of Task2 for the case in which Task2 prebottleneck completes when the $i$ th keystroke is using the bottleneck, which is given by the following:

$$
Q T_{i}^{(n)}=\sum_{k=i}^{n} I K S I_{i}-d_{i}^{(n)},
$$

where $d_{i}^{(n)}$ stands for the portion of the Task2 prebottleneck processes that overlap the bottleneck process of the $i$ th keystroke of an $n$-letter word; that is,

$$
d_{i}^{(n)}=\left(T_{2}+S O A\right)-\left(T_{1}+\sum_{k=1}^{i-1} I K S I_{k}\right),
$$

with SOA being stimulus onset asynchrony (the interval between onsets of S1 and S2). As noted above, this condition occurs with probability $p_{i}$, and $d_{i}^{(n)}$ is defined only within the range between 0 and $I K S I_{i}$.

Because Task2 prebottleneck is independent of word length, $d_{i}^{(m)}=d_{i}^{(n)}=d_{i}$ for any $m$ and $n$, so for given $i$,

$$
\begin{aligned}
Q T_{i}^{(n)} & =\sum_{k=i}^{n} I K S I_{i}-d_{i} \\
& =\sum_{k=i}^{n-1} I K S I_{i}-d_{i}+I K S I_{n} \\
& =Q T_{i}^{(n-1)}+I K S I_{n} .
\end{aligned}
$$

That is, if $i$ is fixed, the queuing time increases by $I K S I_{n}$ for each increase of the number of letters in a word. However, exceptions are cases in which the Task 2 prebottleneck processes complete before the first keystroke starts the bottleneck (i.e., $i=0$ ) and in which the Task 2 prebottleneck processes complete only after all keystrokes have gone through the bottleneck (i.e., $i>\mathrm{n}$ ). Under these conditions, $Q T_{i}^{(n)}=0$. Specifically, at $i=n+1$, there is as much increase as $I K S I_{n+1}-d_{n+1}$ in $Q T_{n+1}{ }^{(n+1)}$ as compared to
$Q T_{n+1}^{(n)}$. For $i=0$ and $i>n+2$, there is no increase in the queuing time.

To obtain the mean of queuing time over all $i$, we compute the sum:

$$
E\left[Q T^{(n)}\right]=\sum_{i=0}^{N+1} p_{i} \cdot E\left[Q T_{i}^{(n)}\right]=\sum_{i=1}^{n} p_{i} \cdot E\left[Q T_{i}^{(n)}\right] .
$$

From the equation, we have for $n=3$ :

$$
E\left[Q T^{(3)}\right]=\sum_{i=1}^{3} p_{i} \cdot E\left[Q T_{i}^{(3)}\right] .
$$

For $n=4$, we have:

$$
\begin{aligned}
E\left[Q T^{(4)}\right] & =\sum_{i=1}^{4} p_{i} \cdot E\left[Q T_{i}^{(4)}\right] \\
& =\sum_{i=1}^{3} p_{i} \cdot E\left[Q T_{i}^{(3)}+I K S I_{4}\right]+p_{4} \cdot E\left[Q T_{4}^{(4)}\right] \\
& =\sum_{i=1}^{3} p_{i} \cdot E\left[Q T_{i}^{(3)}\right]+\sum_{i=1}^{3} p_{i} \cdot E\left[I K S I_{4}\right] \\
& +p_{4} \cdot E\left[I K S I_{4}-d_{4}\right] \\
& =E\left[Q T^{(3)}\right]+\sum_{i=1}^{4} p_{i} \cdot E\left[I K S I_{4}\right]-p_{4} \cdot E\left[d_{4}\right] .
\end{aligned}
$$

Similarly, for $n=5$, we have:

$$
\begin{aligned}
E\left[Q T^{(5)}\right] & =\sum_{i=1}^{5} p_{i} \cdot E\left[Q T_{i}^{(5)}\right] \\
& =E\left[Q T^{(4)}\right]+\sum_{i=1}^{5} p_{i} \cdot E\left[I K S I_{5}\right]-p_{5} \cdot E\left[d_{5}\right] .
\end{aligned}
$$

Thus, from $n$-letter words to $(n+1)$-letter words, the queuing time increases by:

$$
E\left[\Delta Q T_{\text {unint }}\right]=\sum_{i=1}^{n+1} p_{i} \cdot E\left[I K S I_{n+1}\right]-p_{4} \cdot E\left[d_{n+1}\right],
$$

which is Equation 1 in the main text and corresponds to the increase of the PRP effect if typing is an uninterruptible process. It suggests that the PRP effect increases by IKSI per letter if Task2 prebottleneck always completes after the first keystroke has started the bottleneck process and before the last keystroke of the shorter word finishes it (i.e., $p_{1}+\ldots+p_{n}=1$ ). If this condition is not met, the increase in the PRP effect becomes smaller than IKSI (see footnote 1 in the main text).

\section{Interruptible Typing Case}

Predictions for the interruptible case are illustrated in Figure A3. In contrast to the uninterruptible case, Task 2 is queued only until the keystroke that currently occupies the bottleneck completes the process. Then, the queuing time of Task 2 when it arrives at the $i$ th keystroke of an $n$-letter word is as follows:

$$
Q T_{i}^{(n)}=I K S I_{i}-d_{i}
$$

where $d_{i}$ is as defined as in the uninterruptible case. Again, exceptions are when Task 2 arrives at the bottleneck before the first keystroke starts the process (i.e., $i=0$ ) and when Task 2 arrives at the bottleneck after the last keystroke has completed the process (i.e., $i>n$ ), in which cases $Q T_{i}^{(n)}=0$. Importantly, because both $I K S I_{i}$ and $d_{i}$ are independent of $n$, so is $Q T_{i}^{(n)}$. Therefore, in general, the queuing time is given by 


$$
\begin{aligned}
E\left[Q T^{(n)}\right] & =\sum_{i=0}^{N+1} p_{i} \cdot E\left[Q T_{i}^{(n)}\right]=\sum_{i=1}^{n} p_{i} \cdot E\left[Q T_{i}^{(n)}\right] \\
& =\sum_{i=1}^{n} p_{i} \cdot E\left[I K S I_{i}-d_{i}\right] .
\end{aligned}
$$

Consequently, for three-letter words,

$$
E\left[Q T^{(3)}\right]=\sum_{i=1}^{3} p_{i} \cdot E\left[I K S I_{i}-d_{i}\right] .
$$

For four-letter words,

$$
\begin{aligned}
E\left[Q T^{(4)}\right] & =\sum_{i=1}^{4} p_{i} \cdot E\left[I K S I_{i}-d_{i}\right] \\
& =E\left[Q T^{(3)}\right]+p_{4} \cdot E\left[I K S I_{4}-d_{4}\right] .
\end{aligned}
$$

For five-letter words,

$$
\begin{aligned}
E\left[Q T^{(5)}\right] & =\sum_{i=1}^{5} p_{i} \cdot E\left[I K S I_{i}-d_{i}\right] \\
& =E\left[Q T^{(4)}\right]+p_{5} \cdot E\left[I K S I_{5}-d_{5}\right] .
\end{aligned}
$$

Thus, from $n$-letter words to $(n+1)$-letter words, the queuing time increases by the following:

$$
E\left[\Delta Q T_{i n t}\right]=p_{n+1} \cdot E\left[I K S I_{n+1}-d_{n+1}\right],
$$

which is Equation 2 in the main text and corresponds to the increase of the PRP effect if typing is an interruptible process. It shows that the increase of the PRP effect per letter should be smaller than IKSI unless $p_{\mathrm{n}+1}=1$ (i.e., Task2 prebottleneck processes always complete after the last keystroke of the shorter word and before the last keystroke of the longer word) and $E\left[d_{\mathrm{n}+1}\right]=0$ (i.e., Task 2 prebottleneck processes always complete at the same time as the completion of the bottleneck process for the last keystroke of the shorter word). These two conditions are unlikely to be satisfied. On the other hand, the equation shows that there might be no increase in the PRP effect if Task 2 prebottleneck always completes before the last keystroke (i.e., $p_{\mathrm{n}+1}=0$ ). Otherwise, the increase should be greater than zero and smaller than IKSI.

Received May 30, 2012

Revision received August 16, 2012

Accepted August 17, 2012 